\title{
Implications of Smartphone User Privacy Leakage from the Advertiser's Perspective
}

\author{
Yan Wang ${ }^{\mathrm{a}, 1}$, Yingying Chen ${ }^{\mathrm{b}}$, Fan Ye ${ }^{\mathrm{c}}$, Hongbo Liu ${ }^{\mathrm{d}, 1}, \mathrm{Jie}^{\cdot} \cdot \mathrm{ng}^{\mathrm{e}}$ \\ ${ }^{a}$ Department of Computer Science, Binghamton University, 4400 Vestal Parkwa न न st bu. rhamton, NY 13902 \\ ${ }^{b}$ WINLAB, Rutgers University, 671 Route 1 North Brunswi $\kappa, N^{\prime}$ - 9092 \\ ${ }^{c}$ Department of Electrical and Computer Engineering, Stony Brook Universii, 10 Engineering Bldg., Stony \\ Brook, NY 11794 \\ ${ }^{d}$ Department of Computer Information and Technology, IUPUI, 799 West $\Lambda^{\circ}{ }^{\circ}$ chigan Street, ET 301 \\ Indianapolis, IN 46202 \\ ${ }^{e}$ Department of Computer Science, Florida State Universitu, 1 "Iahr see, FL, 32306
}

\begin{abstract}
Many smartphone apps routinely gather various - ivate 1 ser data and send them to advertisers. Despite recent study on protection mechanı. ' $`$ and analysis on apps' behavior, the understanding about the consequences of such priv ${ }^{2} \mathrm{v}$ losses remains limited. In this paper, we investigate how much an advertiser can infer a " "sers' social and community relationships. After one month's user study involving abrit 1s most popular Android apps, we find that an advertiser can infer $90 \%$ of the social relai on 'ips. We further propose a privacy leakage inference framework and use real mobility 'races and Foursquare data to quantify the consequences of privacy leakage. We find that achieving $90 \%$ inference accuracy of the social and community relationships requires ıerely : weeks' user data. Finally, we present a real-time privacy leakage visualization toc that $u$, tures and displays the spatial-temporal characteristics of the leakages. The discu ar: is $v$ iderscore the importance of early adoption of privacy protection mechanisms.
\end{abstract}

Keywords:

Smartphone, Privacy, s sia Relationship

\section{Introduction}

The huge su cess $\left(^{f}\right.$ smartphones is largely fueled by the availability of millions of phone apps that provia func ions covering all aspects of our lives. A large portion of these apps are free. Their levelop rs get financial support from advertisers by embedding their advertisement libraries to $a_{*} n{ }^{n}$, mobile advertisements to users. Many advertisers exist and some of the

Email a $a_{\mathrm{c}}$ esses: yanwang@binghamton.edu (Yan Wang), yingche@scarletmail.rutgers.edu (Yingying Chen), fan.ye@stonybrook.edu (Fan Ye), hl45@iupui.edu (Hongbo Liu), jyang5@fsu.edu (Jie Yang)

${ }^{1}$ This work was conducted during his Ph.D. study at Stevens Institute of Technology. 
major players include Google, DoubleClick and AdMob [1, 2]. To gain better 'nderstanding of user habits and behaviors for accurate ad targeting, these apps customarilv scave_ze private user data, ranging from the phone's IMEI number, MAC addresses of rear y access points, the user's location, even the contact list, and send it to advertisers [ $\bullet 4]$. ' ${ }^{\top}$ timately, these "free" apps are not entirely free: users pay the price of their privac.

There has been quite some recent work that investigates the $\mathrm{pr} \cdots \mathrm{y}$ leakage and potential defense mechanisms. TaintDroid [3] can track the flow of differf at kin 's of private information (e.g., IMEI, location) within an app and log the leaking of sucı infor ration through network interfaces. Barrera et al. [5] and Felt et al. [6] examined pf mis ic 's requested by about 1,000 apps and found requests for unnecessary permissions cor monly e ist. A number of tools $[7,8]$ can help users manage permissions granted to apps such t t th they do not have access to certain private information. Sowayway [9] and Kir $\left\lceil 10_{1} \ldots\right.$ n detect over-privileged apps or identify requests of dangerous combinations of $\mathrm{p}$ - . . . Bunu. Beresford et al. and Zhou et al. proposed MockDroid [11] and TISSA [12] respertively $\cdots$ obfuscate private information so that adversaries only receive empty, fake or anonym ${ }_{2}$ or information. Agarwal et al. [13] proposed a crowdsourcing based mechanism to help us $x_{\sim}$ recide proper privacy settings.

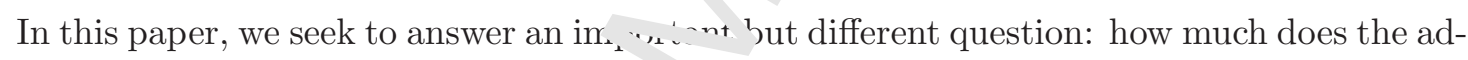
vertiser know about the user, in particular, her social and community relationship (e.g.,family, colleagues and friends) from the le ked 1 -ivate data? This is motivated by a couple observations. First, there is only limitea 'tur $y$ of apps' dynamic leakage behavior at run-time. Existing study $\left[14,9,4,11,15125, \digamma^{7}\right.$ is mostly on the static aspects of apps' permissions. TaintDroid [3] can be used $\uparrow, \log u_{\text {- }}$ - leaking activities but the paper did not focus on a systematic study on the dest.natiu - frequencies and types of apps' run-time privacy leakages. Second, the consequenc's ol such leakage, especially when an advertiser gathers such private data from many users anu cross many apps, is not known either. It is easy to conjecture that the advertiser may ain $\mathrm{ddd}^{\text {i }}$ ional information when cross-examining private data, but exactly what can be lear ${ }^{r^{+}}$remı: is an open issue.

We focus or one im rortant aspect of that perspective, the social and community relationships of a $u_{\sim}$, sucin as her family, colleagues and friends. Such knowledge is an important channel for the ad ertiser to push relevant advertisements since people tend to take note on things $t^{\prime} \quad{ }_{n}$ acquaintances have done (e.g., bought). For example Facebook has largely relied on people sluntarily publicizing such relationship. However, many real world relationships are not publicized online yet they are equally important to advertisers; and there is a trend for 
Facebook users of various age groups to go for other "small-circle" social net orks, or become less and less active due to privacy concerns $[16,17]$.

In particular, we quantify to what extent an advertiser can learn an inf : users' relationships by developing a privacy leakage inference framework. Our syste. atic ' ' 1 ddy on privacy leakage inference involves both real experiments with multiple volunt er as wtll as trace-driven studies with human mobility traces obtained from two data sets, na. ${ }^{\circ}$ y MIT reality trace [18] and Foursquare trace [19]. By examining the privacy leakages of pa ticipants from a diverse background ranging from academia to city environments (i.e., vur of $\mathrm{ll}$ experiments and the MIT trace are academia whereas the Foursquare trace rep eser city environment), we discover that the privacy leakage enables an advertiser to in ` a sion: .1cant portion of a user's real world relationships that have physical interactions. Our prive sy leakage model and inference

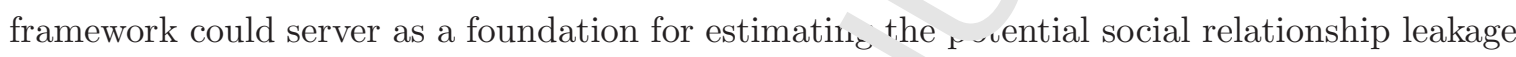
of a particular user based on his/her app usage.

Specifically, we make the following contributions:

- We conduct a manual study of the fr^quen es, destinations and types of the run-time privacy leakages of nearly 200 most po $_{\perp}$ uı apps across 19 categories in Google Play. We discover that major advertisers ${ }^{n} \mathrm{n}$ eavily gather all types of private data in short time from many users.

- We model the relationship $\mathrm{i}_{\Lambda_{\text {. }}}{ }^{\prime}$ rence process in a three-layer framework and define the concept of connection, w'ich is exemplified by two users sharing similar patterns in their leaked data (e.g.. con nor Wi-Fi access points). We conduct a one-month real experiment of $10 \mathrm{par}^{+} \mathrm{C}_{\mathrm{c}_{1}}{ }^{a} \mathrm{nts}$ of family, colleague and friend relationships, using various apps in their daily 1 . s. We find that by aggregating data across users and apps, an advertiser can inı $n$ er $90 \%$ of the relationships from the "connections".

- We further pr por $\mathrm{t}$ tw models for users' temporal privacy leakage profiles based on the experiment - stua Гo verify the generality of findings from the real experiments based on privacy leakag inference, we conduct trace-driven studies by populating the derived user $\mathrm{p}$ viles to the human mobility traces in the MIT reality [18] and the Foursquare datase ${ }^{+s}$ [19]. We find that the advertiser can infer $80-95 \%$ of a regular user's relation in ac 1 mir and city environments after gathering only 3 weeks of private data.

- Finall, we build a visualization tool that captures and displays the spatial-temporal statistics of different types of privacy leakage on both per app and per destination basis 


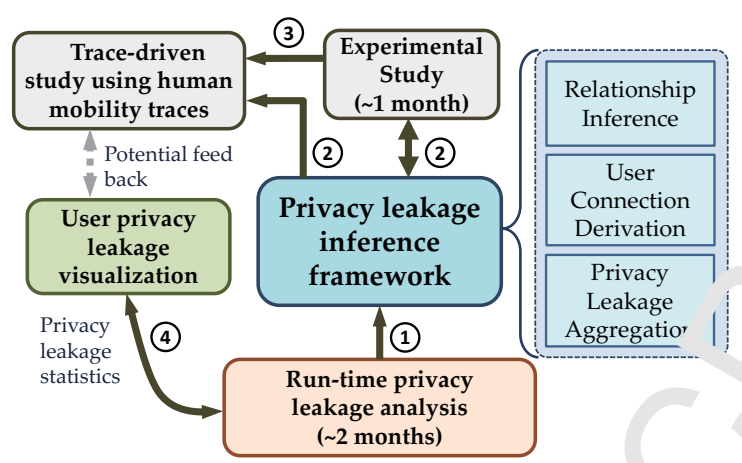

Figure 1: We take a four-step approach to understand the advertiser's pernecu._. _. users' social and community relationships.

in real time, which helps users gain better insights $~ n$ tic scope and degree of privacy losses. This tool can serve as a complement n recor mendation-based user privacy protection mechanisms [13].

\section{Approach Overview}

To facilitate the understanding on the consequ aces of privacy leakages, we take a four-step approach: run-time privacy leakage study, pr rav-leakage inference and profile modeling via experimental study, inference framework valuavion via trace-driven study, and user privacy leakage visualization, as depicted in Fiøure 1.

From the advertiser's perspecti e, we s udy two types of relations: social relationship and social community. The social re ations.

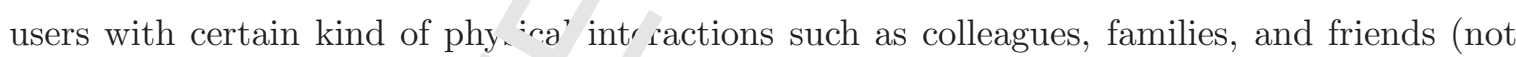
virtual friends from online s cial networks). Whereas a social community involves more than two users, who usually appear at - location during the same time period for certain common interests. For example a $g$ oup of students taking the same class twice every week or people eating in the sar e resu rant every Tuesday. We believe identification of both kinds of relationships help a 've cise $s$ design better targeted advertising strategies.

Run-Time rivacy Leakage Study. First we want to systematically understand the destinations, fre ruencif; and types of the leaking behavior of apps to understand the flow of common pr stice of privacy leakages in apps. We study their spatial and temporal privacy leakage cha cteris ics to complement the existing work, which focuses on static aspects of permiss $\because$ us 7,20$]$, or provides the capability of logging the leakage but stops short of a systemat study [3]. The Wall Street Journal (WSJ) study [21] in 2010 investigated the types of leakages for about 50 most popular apps, but not destinations and frequencies, and 
it was a bit outdated given the fast pace of the mobile market. Thus we cc 'duct a series of experiments over a about two-month period to obtain the most up-to-date picture of privacy leakages, where we also find changes in the types of leakages for about hr if of the apps studied in $[21]$.

Privacy Leakage Modeling. To understand the consequence or privacy leakages when an advertiser combines the data received from different users, we at ${ }^{\prime}>\mathrm{p}$ a three-layer privacy leakage inference framework (as depicted in Figure 1) includin, Priv $c y$ Leakage Aggregation, User Connection Derivation and Relation Inference.

We introduce an important concept connection, which $\mathrm{x}$ xist w'en two users share similarities in leaked data. The connection helps bridge the gap 'otween aw privacy leakage data and higher level relationship inference. The intuition is that eacı type of particular relationship has certain temporal-spatial patterns in users' physic 1 inu ....tions, which can be captured by connection. For example, two family members us, " n w w together at home during late night and early morning; while classmates encounter each ou. $\rightarrow$ f frequently in classrooms during the daytime of weekdays. Although exceptions to $\mathrm{su} \mathrm{h}$ patterns exist, it can usually identify most relationships and is the standard practice wid ' $y$ - dopted in social community inference [22, 23]. We conduct an additional experimental an in 10 participants for over one-month time period to confirm the effectiveness of our privacy leakage model, which also reveal that the temporal-spatial similarities betwef 1 peo $_{\mathfrak{1}}{ }^{\prime} \mathrm{e}$ who have friend relationship is not as regular as that between people of other relation hir, that have repetitive interactions (e.g., colleagues and families).

Evaluation of User Pr vacy - erence. To understand whether the above observations can be generalized to largur sci. user population with various backgrounds, we extract user privacy leakage profiles. app y them to user mobility traces generated from two datasets with over 500 participants $W$ verify that using 3 weeks of private data, an advertiser can infer colleague-based rels ion hip of regular users at around 90\% accuracy in an academia environment, and friend asea - ationships above $95 \%$ in a city environment. When an advertiser uses hierarchica cluster ng to infer social communities, $80-90 \%$ of those of regular users' are revealed in s calemıa environments, and over $80 \%$ in a city environment.

User F ivacy Leakage Visualization. Finally, we believe that explicit presentation of privacy raoes to users will help them gain better understanding of the privacy loss. As a starting st , we leverage TaintDroid [3] to develop a tool that visualizes the temporal and spatial characteristics of the leakages in real-time on a mobile device. We note that the privacy 
Table 1: Counts of six types of frequent privacy leakages to top-five appeared destina ${ }^{\dagger} u_{-}$during one day (including three time periods) testing for each app.

\begin{tabular}{|c|l|l|l|l|l|l|}
\hline Destination & Contact & $\begin{array}{l}\text { Phone } \\
\text { Number }\end{array}$ & IMEI & GPS Location & $\begin{array}{l}\text { Network-based Lo- } \\
\text { cation }\end{array}$ & elerometer \\
\hline Google & 70 & 75 & 49 & 90 & 110 \\
\hline DoubleClick & 5 & 6 & 3 & 9 & 11 & 3 \\
\hline Mixpanel & 0 & 0 & 0 & 2 & 9 \\
\hline Flurry & 2 & 2 & 0 & 4 & 7 \\
\hline Amobee & 0 & 0 & 6 & 0 & 4 \\
\hline
\end{tabular}

leakage inference model together with the privacy leakage dat can rotentially augment the visualization tool so that users can see the consequences on + ${ }^{*}: r$ relationship in real-time, which hopefully can guide their usage of apps (e.g., stor using $\mathrm{Y}_{\lrcorner p}$ for the rest of the day or resetting the ad tracking ID once a threshold of relationships re exposed).

\section{Run-Time Privacy Leakage Study}

Starting in Android API level 23, users can graı ${ }^{-}$permissions to apps even when they are running. App permissions could be manually eve - ' by users, and unauthorized communications among apps are prohibited. Althor $h$ the permission control mechanism seem to be friendly to users, they are not effective in pro ecung users from malicious apps that request to collect data irrelevant to the main funci $\urcorner n$ of the app. Moreover, once the application is granted access, the OS does not har- $f_{1}$ rrther control on when a type of private data is accessed and how it is used by the $c$. nlicati $n$ at run-time. The application is free to access it as frequently as possible and ser $d$ it to $w$ nerever it wants over the Internet $[3,9,1]$.

The most similar study to $c^{\prime \prime} s$ is he WSJ one [21] in 2010, which focuses on 5 privacy leakage types (i.e., contact, 'ncation, phone id, and phone number) among 50 most popular apps from Google Play. Tro results are interesting but there is no analysis on the frequencies and destinations of pris ' $c y$ ' Jakages. Our study aims to provide a more comprehensive and upto-date analysis incl din , the irequencies and destinations of the leakages. We also investigate how much private $\mathrm{d}_{\mathrm{a}}$ - an dvertiser can collect and aggregate from multiple apps. This helps the user unders ${ }^{\dagger}$ nd th $>$ scope and extent of privacy leakages when running apps; it also serves as the basis for $\mathrm{i}$ for alation of the privacy leakage inference in the next section.

\subsection{Metho' 'ology}

We chnose ure top 10 most popularly downloaded applications from each of the 19 categories in Google Fiay as of January 2013, totaling 190 applications. We expect that these most popularly downloaded apps are installed by the majority of users, thus their behavior analysis is 
representative to the majority of users. Our app behavior study is grounded c . TaintDroid [3], which helps to track and log the privacy leakages of the applications. Once an .pplication accesses private data, TaintDroid generates a taint log in the Android s ster log.

We use an Android application called CatLog to capture the system ' ’o an.' save the leaked information as a text file in the smartphone's memory card. The f slu vings are recorded for each leaking event: application name, the leaked private data, timı $r$ the leakage, the destination to which the data is sent, the event/operation triggerir s the 'akage, and an optional memo. The saved information is downloaded to a computer to in offli e processing, which includes discriminating privacy leakages and calculating the s ${ }^{s}$ equ $n \mathrm{v}$ of privacy leakages. Some of the apps crash during the test, and we are able to gat her com rete results for 145 apps.

We use Google Nexus One and Google Nexus S, hoth run ing Android OS 2.3.4, and the whole study lasts for about two months. To captu the t.pplication's behavior at different times in a day, we test about 4-5 applications ir $1 . . . .4$ unferent time periods (i.e., morning, noon and night) in each day. During each perind, the slected applications are tested one at a time: we first reboot the device to make sure. 11 cached information is cleaned up; then we install the application and perform normal $u x_{5}$ for about 5 minutes; finally we uninstall the application and reboot the device to tes. no $\mathrm{t}$ application. This procedure helps to avoid interference between applications.

\subsection{Findings}

Per App Privacy Leakag .. Dur. ; our study, we find that about $50 \%$ of the apps in the WSJ report have changed he $\mathrm{s}$ pr vacy leakage behavior. In particular, we find that 97 out of the 145 applications r nd out private data of the user. The data includes GPS location, network-based location (prnvided oy Android based on cellular ID and Wi-Fi networks), WiFi Access Point SSID list cor act list, phone number, International Mobile Station Equipment Identity (IMEI), accf erome $\cdot$ readings, but not those of microphone, camera and text message $\log$, which are acce or by ne applications but not leaked out. We also find that there are 8 applications sen ang Wi-r i SSID list (scanned by the smartphone) through SSL, and 3 of the 8 applications ( $\mathrm{s}$., Co .pass, CNN App, Yelp) send this information to the same destination (with IP ad uress 773.194 .73 .104 belonging to Google according to www.iplocation.net). This type of WiF . AP 1*st is most likely the company's effort to build a WiFi address database for geo-loca : on $\ldots$ - poses [24]. It could be employed to infer the user's location, thus potentially her mobility pattern during a day.

. Per Destination Privacy Leakage. We further investigate how the private data 


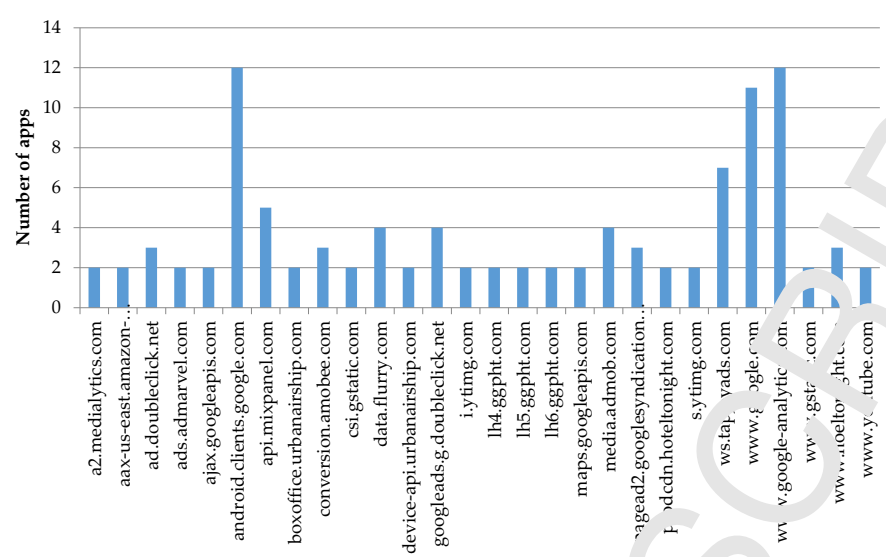

Figure 2: Destinations that collect private data frow. more han one app.

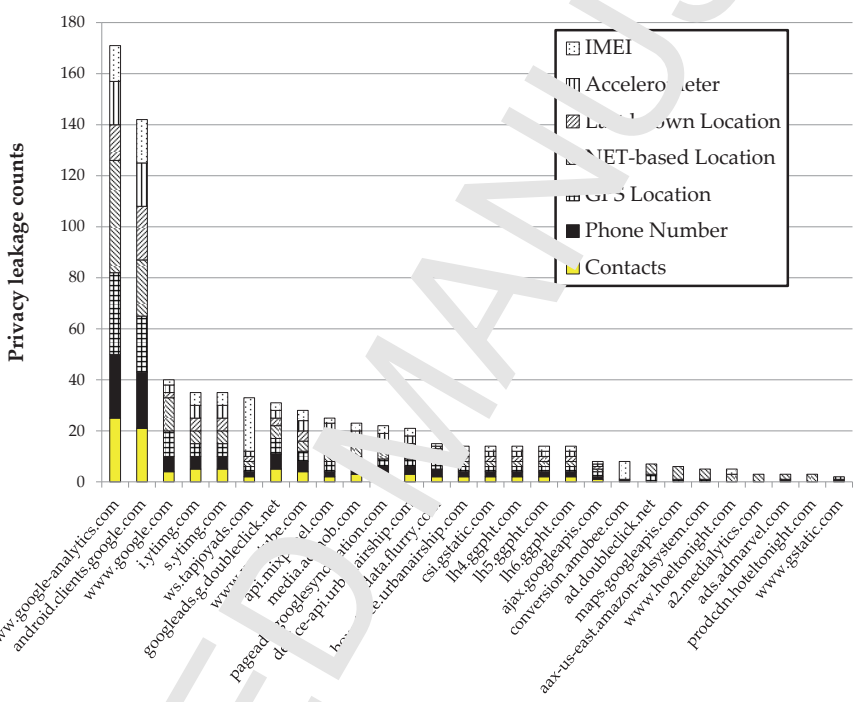

Figure 3: Privacy leakage connts des nations that collect private data from more than one app.

could be collected by a sin', te Jestination (e.g., an advertiser's server) through multiple apps. Table 1 summarizes the a aber of times that 6 types of private data are leaked to the top 5 most frequent destinatic 4 . with one day test for each app. It is not surprising that Google and DoubleClick (bough ${ }^{+}$by Googı in 2008) dwarf the other three much smaller players due to their dominance to the mo 'o a vertising market. We also observe that the location information is the most freque it leak $\mathrm{d}$ type, followed by phone number and contact list.

We show the doct nations receiving data from more than one app in Figure 2 (called "common" lestina ions). We identify 28 such destinations in 19 categories. Specifically, we find that thre $\sim$ uogle destinations collect private data from more than 10 applications, and www.tap, $\mathrm{y}$ ds.com from 7 applications. Among all 97 applications showing privacy leakages, 26 of them su 7 different types of privacy data to Google, while 9 applications send 7 differ- 


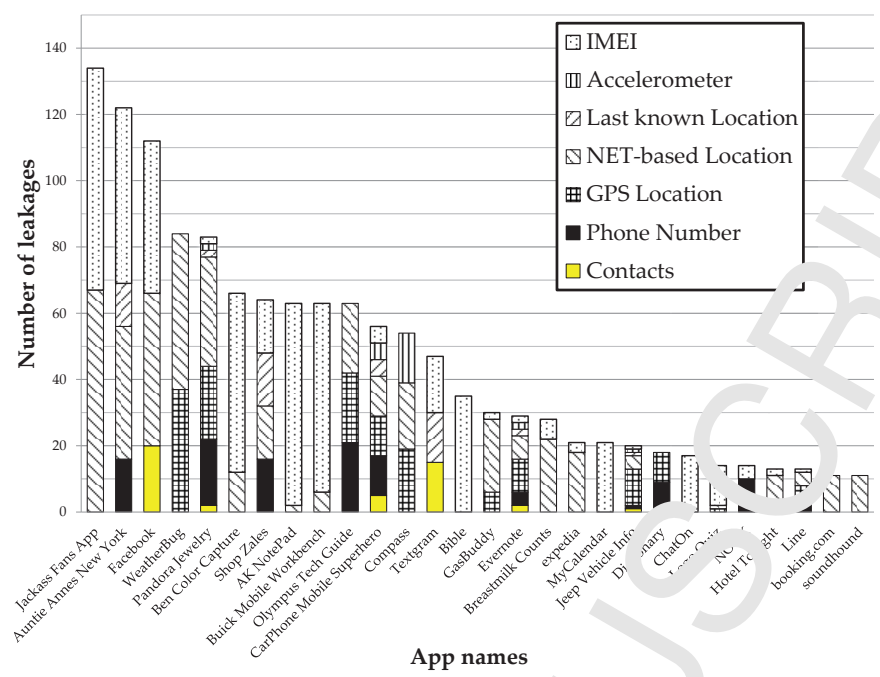

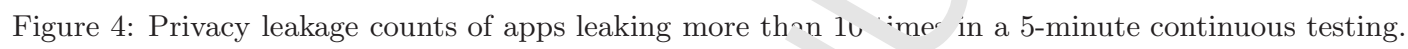

ent types to DoubleClick. Figure 3 shows that durine nnn 'ay's testing of the 97 applications, most of the 28 destinations collect more than 3 types $f$ private data, and Google is the most active one among these destinations.

Furthermore, we find that among applica ‘, s se. ding to common destinations, they usually send out different types of private data For in tance, we observe that app Pandora Jewelry sends out IMEI, phone number and contact lis. whereas app Evernote sends out phone number and location information. This cor . $\mathrm{rm}_{\infty}$ our conjecture that an advertiser can combine the private data from different apps to $s$ in a nore complete picture of the user's behavior.

Privacy Leakage Frequer cy. Juring our testing, we find that the Location and IMEI are the first and second most cor mon, riv acy leakage types, which involves 71 and 61 applications respectively. We present $t^{l}$ e $l^{1}$. 'rage count decomposition of 28 apps that leaks more than 10 times in its 5-minute $\mathrm{u}_{\mathrm{ag}}$ in Figure 4 . We observe that at least three different types of private information are $\ldots$ ed for most of these apps. Specifically, the combination of IMEI and NET-based locr ion is the most leaked information. We also notice that both Facebook and Textgram have ab at, 20 times leakages of address book when the user queries his friends in the social ne work.

These res.1t $\mathrm{s}+\cdots$. data (such is IME and NET-based location) to identify the location of the user from such apps, and furtıc obtain a fine-grained picture of the user's social life with the assistance of the leake $r$ sntact information. Additionally, we observe that 7 apps shown in Figure 4 have leaked more than 4 types of private information, 3 of them have even leaked all 7 types of 


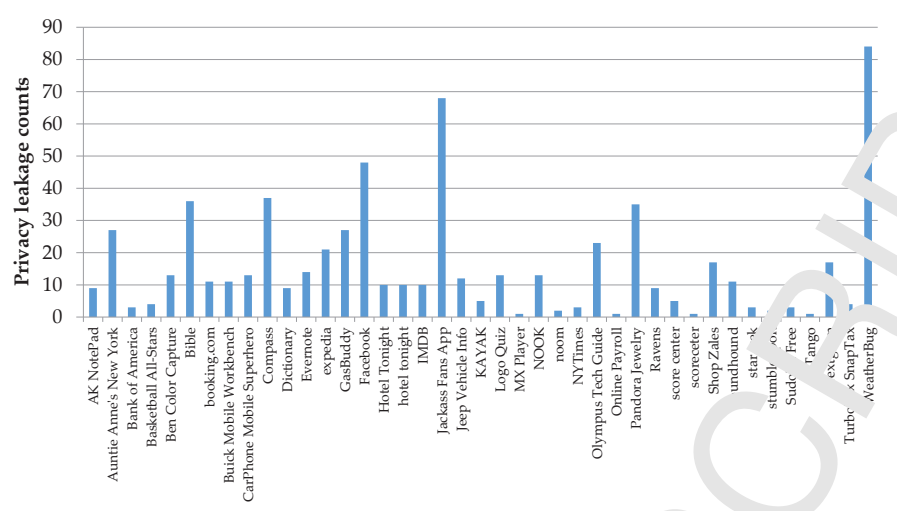

Figure 5: App leakage frequency to destination study: top 41 apps $\mathrm{r}$ ivine cu 'rages to multiple common destinations in 5-minute usage for each app.

private information (i.e., Pandora Jewelry, Carphone Mobile 'uperhero, and Evernote). This equips the advertiser with a comprehensive view of $\therefore$ us $\cdots$ private data through their daily phone usage.

We also find that it is common for different applica ions to have similar combinations and frequencies of privacy leakages. For example, Ga. B ^ddy and Breastmilk Counts have the same number of Net-based location and IMEI leaka, ${ }^{2}$. in the 5-minute usage. Such applications have

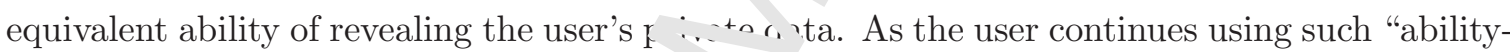
equivalent" apps, the leaked private data $\operatorname{kee}_{\mathrm{p}} \mathrm{s}$ at similar levels all the time. The situation becomes more severe when these ar plicau nns send the private data to the same destination.

Leakage to Common Destina. 'ns We take a closer look at the leakage frequency from different apps to a "common" d stir ation. We first show the number of privacy leakages for the top 41 apps sending to comm on de ir ations in a 5-minute usage in Figure 5. We observe that WeatherBug and Jackass F ins a $\curvearrowright$ the top two apps with the most frequent leakages: they leak about 80/70 times durir g t e 5 minute period. We further summarize the leakage frequency in app categories to comı. $\eta$ destinations and observe that the Weather category exhibits the highest privacy leak age f equency, partly due to their needs to know the user's location, and the Social category is the su $\mathrm{nd}$. This again confirms that various apps leak private information to multiple com non de inations, which allows the advertiser to piece together the user's social picture at fir empual granularity through multiple apps.

\section{User Pi vacy Leakage Modeling and Experimental Study}

In th: : sf iuvn, we present a privacy leakage inference framework that quantifies to what extent an au 'ertiser can learn and infer users' relationships. We then run real experiments with multiple participants to analyze the consequences of the privacy leakage from the advertiser's 
perspective and abstract privacy leakage user profiles based on the experime ${ }^{\text {ts }}$.

\subsection{Privacy Leakage Modeling}

We first define the concept of connection. A connection between $i$, n sers exists if the same type of privacy leakage from the two users share certain spatial, 'smpural or content similarities. A few examples are:

Contact list: A connection instance exists between two users it hey are in each other's contact list, or they share common contacts. (However, we lote th $t$ contact lists are not sufficient for social relationship inference simply a person dnes +.+ tecessarily to have close relationship with anyone in his or her contact list.)

Wi-Fi Access Point list: A connection instance exists atwuen two users when they share common leaked access points at the same time.

GPS location: A connection instance exists betwee.. ${ }^{+}$wo users if two GPS locations leaked around the same time are close by within a certal.. +hreshold.

Network-based location: A connection in mexists between two users if the leaked network-based locations are close by within э cer . in threshold around the same time.

The connection bridges the gap between he rivacy leakage information and the users' relationship inference. In particular, to qu ntiry che consequences of the privacy leakage from the advertiser's perspective, we design a privacy leakage inference framework, which consists of three virtual layers: Privacy Le kage A gregation, User Connection Derivation, and Relationship Inference as shown in $\mathrm{F}$.gure $\mathrm{u}$.

Such a framework facilitates 's o pr iform a systematic study to understand the advertiser's perspective of user privacy (1) The Privacy Leakage Aggregation layer deals with the raw privacy leakage information. An auvertiser can combine the privacy leakage data from multiple apps across different $\mathrm{u}$ ers ver time. For example, the users can be identified by the IMEI or phone number. T'e agg ${ }_{\perp}$,ated privacy leakage data of each user can then be categorized into different types, 1 n a contact list, AP list, GPS location, and Network location. (2) In the User Conne won Derıvation layer, the advertiser correlates the data from different users and identifies cc nectir $n s^{2}$ between any two users. By correlating different types of privacy leakages ac uss th users over time, the connection frequency between any two users can be derived. (3) ' n the Relationship Inference layer, the user's social and community relationships, such as $7 m_{y}$, olleagues and friends, are inferred based on the connections between users.

\footnotetext{
${ }^{2}$ We use "connections" to refer to connection instances later in the paper.
} 


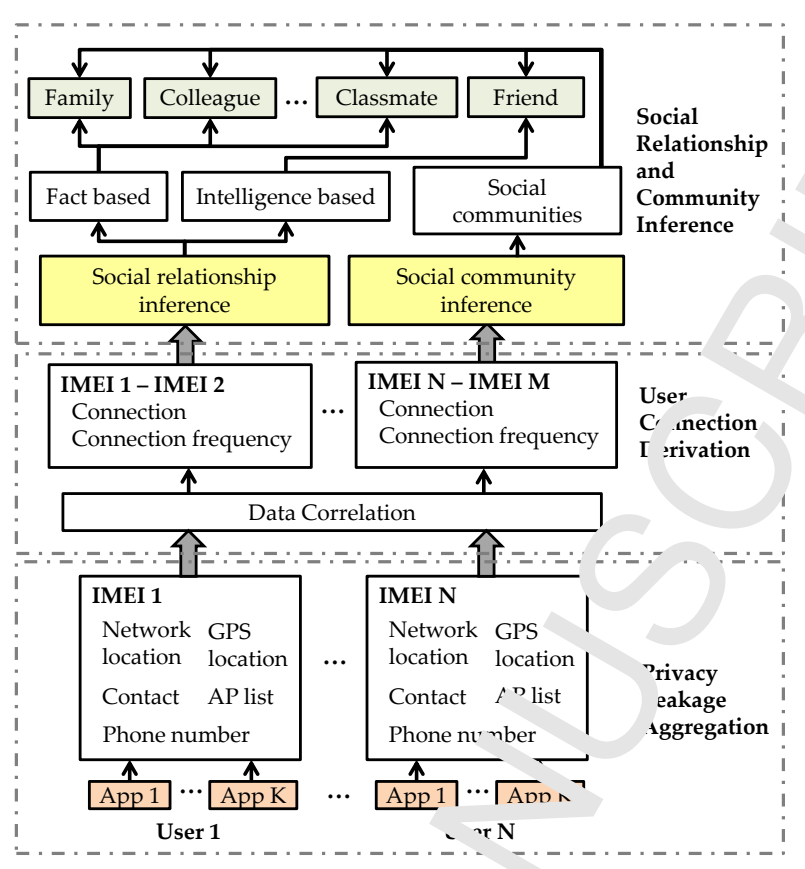

Figure 6: Privacy leakage inference framework with th. of smartphone user privacy.

The type of relationship is usually determinea by xamining the temporal and spacial patterns of the connections (e.g., family members " "laily -lave connections at home in the morning and at night, whereas colleagues have connections in office during working hours). We next conduct an experiment to study the effectiv ness o. our privacy leakage model using this framework.

\subsection{Experimental Study}

\subsubsection{Design of Experiments}

Our experiment involve ${ }^{10}$ volunteer students and their family members over one month period, among which five trnes of relationships exist: colleague, collaborator, classmate, friend, and family. The ten olu ceers are all graduate students between 21 to 24 years. Eight of them major in Coms ter Ens-neering and the other two major in non-computer-related major. All of them have bet. . sing smartphones for over two years and have moderate understandings of information + chnol ngy. To clarify, collaborators are usually colleagues that actively work together, usually at rer alar times such as weekly meetings.

During he $e x_{1}$ riments, we distribute smartphones with our visualization tool (which is presented in Soct; $n$ VI) and the top 10 popular apps across 19 categories in Google Play sending $\sim \mathrm{c}$ smnon destinations (shown in Figure 5) installed. Because the experimental smartphone are not replacements of the volunteers' regular phones, they are asked to use their experimental smartphones at least three times a day. There is no restriction of how and when 


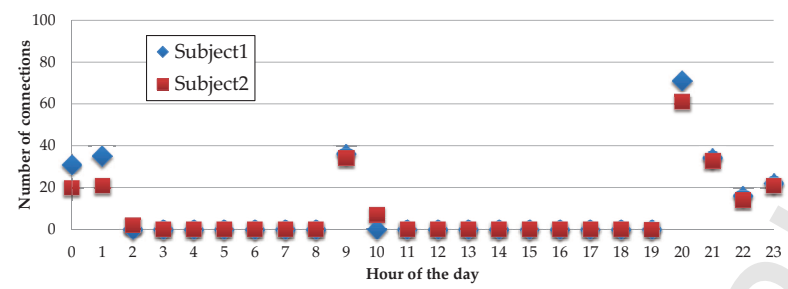

Home, Fact based relationship (family)

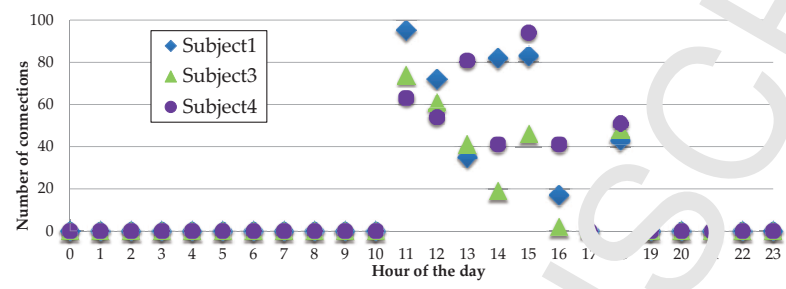

Lab, Fact based relationship (colleag

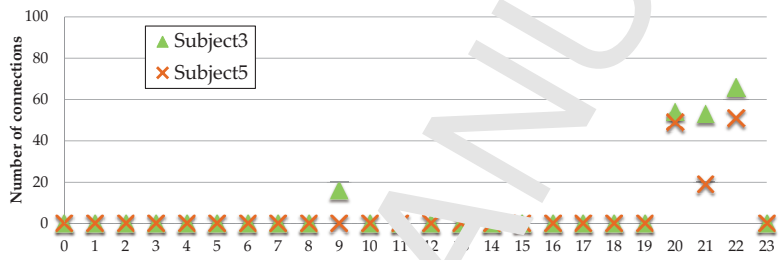

Hour 6 eday

Coffee shop, Intelligenc L. ed l lationship (friend)

Figure 7: Comparison of location leakage patterns if different relationships with respect to locations.

to use the apps in the experiments, and the vlunteers are encouraged to use whichever apps they are interested in without know; $\mathrm{x} \cdot \mathrm{a}$ purpose of this experiment. After the experiments we extract the leaked privacy data ^ rged 1 y our tool to quantify to what extent an advertiser can infer a user's relationships.

\subsubsection{Observations}

User Connection Dorivatiun. Figure 7 shows one example on the temporal patterns of the derived user $\mathrm{co}^{r}$ nect $^{+}$ons at three different locations based on GPS location leakage. We observe obvious s patial a. d temporal patterns of connections corresponding to the ground truth of the social re ${ }^{\prime} t^{\prime}$ ons ${ }^{2}$ ps. In particular, subject1 and subject2 have frequent connections in the morning aroun 1 (UAM) and at night (through 9PM-2AM) at a residential area. The advertiser can t. 'is in in $^{4}$ the two subjects most likely have a family relationship. Further, subject1, s' oject? and subject4 have connections frequently during working hours (through 11AM-7PM, in an sffice building. This follows a typical pattern of colleagues or collaborators. Addition 'lly un connections of subject3 and subject5 usually happen at early night (9PM$10 \mathrm{PM})$ but 'o not show up late night (12AM-6AM) or morning. Such a pattern is more like friends hanging out together. From the above examples, the advertiser can infer users' social 
relationship once it has accumulated enough privacy leakages over time.

Definition of Two Types of Social Relationships. From the experimentaı esults, we observe that while some relationships (e.g., family, colleagues, collabore ors and classmates) exhibit repetitive connection patterns, some others like friends do not. This . because family and colleague based relationships naturally carry similar spatial-tem joı ll patuerns dictated by the relationship. For example, families live together at night whin $~$,lleagues work together during the day, whereas friendship does not necessarily carry such inherent patterns. Two friends that do not hang out for a while are still friends. We a tingr sh these two categories of relationships as Fact Based Relationship and Intelligen e Br , ' Relationship, which covers traditional social relationships. The Fact-based Relatio ship ins' udes colleagues, classmates, roommates, families that carry inherit similar, regular and epetitive spatial-temporal connection patterns as dictated by the relationship, w-reas Intelligence-based Relationship includes friends, which do not necessarily carry s $\ldots$ rwouns.

\subsection{Making Inference based on Thresholding}

We next investigate how accurate an a dvertı r could infer about the user's social relationship such as colleagues, families and frienc by utilizing connections between users derived from different types of privacy leakages.

We use a threshold-based approach to derive relationships based on the connections between users extracted from privac leakage aggregation at the advertisement provider. If the connection count between two $\mathrm{u}$ - rs exc ds a certain threshold in an observation window, we consider some relationship exi - Our framework utilizes the temporal and spatial patterns

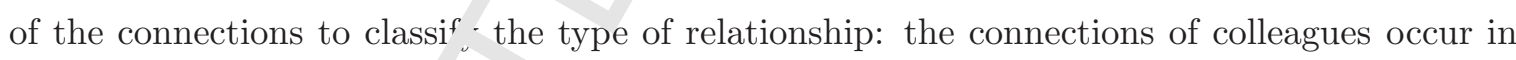
work hours of weekdays, families. .1 early morning and late night, while friends after working time and in weekends.

These simple rulf $;$ may $1, t$ be entirely reliable. Nevertheless, we show that an advertiser can make inference ${ }^{\mathrm{v}} \mathrm{n} \mathrm{v}$, th such simple rules. We note that such temporal and spatial patterns of the connertions are the basis to statistically generate a user's privacy leakage profile, which $\mathrm{w}^{\prime}{ }^{\prime 1}$ be d scribed in Section 4.4.

In total we ha 10 pairs of colleague relationship, 5 pairs of collaborator relationship, 1 pair of famiı. rela ${ }^{+}$onship, 2 pairs of classmates, and 3 pairs of friend relationship among our 10 partı Das u. That is 18 pairs of fact-based relationships and 3 pairs of intelligence-based relationship. During our experiments, we observe that by utilizing connection frequencies and patterns, an advertiser can infer over $90 \%$ social relationship correctly (i.e., all fact based 
relationships and 2 pairs of intelligence based relationship). The only one fr and relationship that is not correctly identified is because this pair of users only have one conneci. on during the testing. This is inline with our expectation since the intelligence-br sed elationship does not carry repetitive spatial-temporal connection patterns, therefore th ${ }^{r}$ con action may not frequently repeat on different days, thus the relationship is harder $+\Omega \mathrm{L}$; idencified.

\subsection{Deriving Privacy Leakage User Profiles}

Based on the experimental data collected over one month, $\mathrm{w}:$ next $\mathrm{L}$ tild the privacy leakage user profile to statistically capture the temporal and spatial nattes we develop two types of privacy leakage user profile, activeness based profile and $p\urcorner \cdot b^{\prime}{ }^{\prime}$ 'lit? based profile, which will be applied to our large-scale trace-driven studies on advertisu 's puspective in the next section.

\subsubsection{Activeness Based Profile}

The activeness based profiles are generated ba $\therefore$ us privacy leakages from each participant in our experiments and aim to capture the fine-orainea satistical view of the privacy leakages.

Step 1. We first derive the privacy leakage $\mathrm{p}$ - jability model of a particular user. Assume there are $N$ types of privacy leakages obser ${ }^{\prime}{ }^{~} \eta$ iotal. We divide the time in day $d$ into $T$ time windows as $\left\{w_{t}, t=1, \cdots, T\right\}$. The $\cdots\left\llcorner h^{\prime}\right.$ a time window $w_{t}$, a vector $\Phi^{u, d, t}$ is defined to capture the numbers of occurrences of diturent privacy leakage types, and each element $\Phi^{u, d, t}(i)(i=1, \cdots, N)$ corresponds $\mathrm{o}$ the umber of times privacy leakage type $i$ occurs. For example, when $\Phi^{u, d, t}$ equals to [2 $1, v_{J}$, it heans 2 occurrences of leakage type 1,1 occurrence of leakage type 2 and 0 occurr nce sf le akage type 3 in time window $W_{t}$ at day $d$ for user $u$.

We then define $\gamma_{i}^{u, d, t}$ to ; idicate - hether the privacy leakage type $i$ appears in the vector $\Phi^{u, d, t}$ as:

$$
\gamma_{i}^{u, d, t}=\left\{\begin{array}{l}
1, \Phi^{u, d, t}(i) \neq 0 \\
0, \Phi^{u, d, t}(i)=0 .
\end{array}\right.
$$

The probability that tyse $i$ leakage happens for user $u$ in time window $w_{t}$ across $D$ days (e.g., $D=7$ days is dt. $\mathrm{n}$ d as:

$$
\operatorname{Prob}_{i}^{u, t}=\frac{\sum_{d=1}^{D} \gamma_{i}^{u, d, t}}{D}
$$

Step 2. The $\mathrm{n}$ ،mber of occurrence of the privacy leakages affects the inference of a user's social ce ritn, ity. Thus we capture the frequency of type $i$ privacy leakage using the average number of $c$ currences over the days it happens in time window $w_{t}$ across $D$ days. Specifically, 
the average rate $r_{i}^{u, t}$ is defined as:

$$
r_{i}^{u, t}=\frac{\sum_{d=1}^{D} \Phi^{u, d, t}(i)}{\sum_{d=1}^{D} \gamma_{i}^{u, d, t}} .
$$

The activeness based profile of user $u$ consists of $\operatorname{Prob}_{i}^{u, t}$ and $r_{i}^{u, t}$.

Example. We illustrate the generation of the activeness based rofi $s$ iser $u$ in Figure 8. We examine a privacy leakage dataset across 7 days (i.e., one week, with the time window $w_{t}$ set to 5 minutes and 288 time windows in total per da. Ass me 3 types of privacy leakage are under study. For $w_{t}$ at day 2, if there are 2 ocmeren of leakage type 2 and 8 occurrences of type 3 observed, we have $\Phi^{u, 2, t}=[0,2,8] \mathrm{zn}^{\prime} \gamma_{2}^{u, ?, t}=1$ shown as the green eclipse in Figure 8. In addition, if the leakage type 2 is $\mathrm{c}^{{ }^{1} \mathrm{~V}}$ unserved during day 1 and day 2 with $\Phi^{u, 1, t}=[0,5,7]$ and $\Phi^{u, 2, t}=[0,2,8]$, the priva - leaka, e probability of type 2 privacy leakage in time window $w_{t}$ across 7 days for user $u \iota$ n be calculated using Equation 2 as: $\operatorname{Prob}_{2}^{u, t}=\frac{\gamma_{2}^{u, 1, t}+\gamma_{2}^{u, 2, t}+\cdots+\gamma_{2}^{u, 7, t}}{7}=\frac{1+1+\cdots+0}{7}=0.2 \mho_{\iota}$ And the corresponding average rate can be obtained as: $r_{2}^{u, t}=\frac{\Phi_{2}^{u, 1}+\Phi_{2}^{u, 2}+\cdots+\Phi_{2}^{u, 7}}{\gamma_{2}^{u, 1, t}+\gamma_{2}^{u, 2, t}+\cdots+\gamma_{2}^{u, 7, t}}=\frac{5-1+\cdots+0}{1+\cdots}=3.5$, which is shown in blue rectangles in Figure 8.

Categorization. Once the activeness bas ' $d$, ser profile is obtained, the advertiser could further categorize the profiles by the num ${ }^{\wedge} r$ ol nours $k_{u}$ the user $u$ has privacy leakages in a one-day duration. There are three renresentative user categories, namely active user category, regular user category, and inactive user cetegory. Assume two thresholding hours $\rho_{1}$ and $\rho_{2}$ with $\rho_{1}>\rho_{2}$. If the user $u$ has greate ' nan $\rho_{1}$ hours with privacy leakages, his user profile is put into the active user cate ${ }^{\prime r}$. If he user $u$ has less than $\rho_{1}$ but larger than or equal to $\rho_{2}$ hours with privacy leake ^s, his user profile is then added into the regular user category. When the user $u$ has less than $\rho_{2}$ hours privacy leakages, his user profile is then captured in the inactive user categr :y. $r$ he categorization can be summarized as:

$$
=\left\{\begin{array}{l}
1 \text { (active user category), if } k_{u} \geqslant \rho_{1} ; \\
2 \text { (regular user category), if } \rho_{2} \leqslant k_{u}<\rho_{1} \\
3 \text { (inactive user category), if } k_{u}<\rho_{2} .
\end{array}\right.
$$

\subsubsection{Prob bility Based Profile}

The $\left\{\operatorname{Pr}_{c},{ }^{u, t}{ }_{\imath}, t\right\}$ in each activeness based profile captures the leakage of the corresponding user $u$. ' ' $\mathrm{cr}$ dı cucterize the statistical average of the leakages of users in the same category, we design the, obability based profile. Activeness based profiles in the same category are used to derive the leakage probability and rate for the probability based profile of that category. 


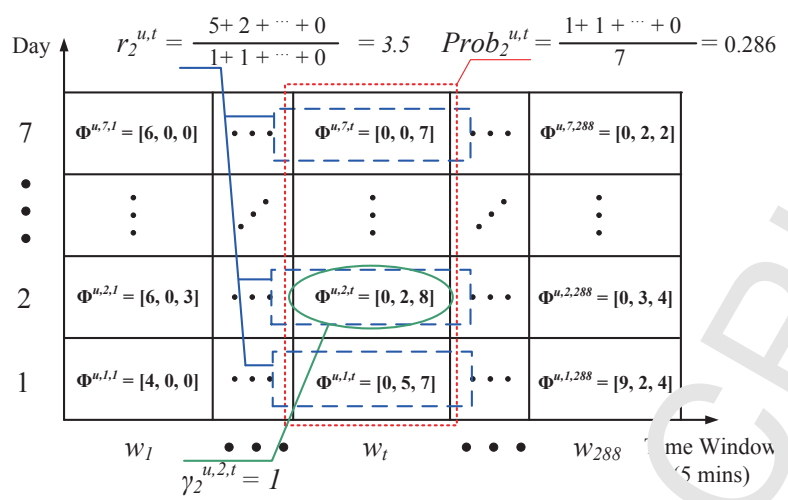

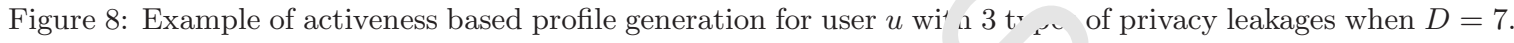

Step 1. We first define the $\delta_{i}^{u, t}$ to indicate whether are i probability of type $i$ leakage in the time window $w_{t}$ for the profile of user $u$ as:

$$
\delta_{i}^{u, t}=\left\{\begin{array}{l}
1, \operatorname{Prob}_{i}^{u, t} \neq^{n} \\
0, \operatorname{Prou}_{\imath}^{u, t}=0 .
\end{array}\right.
$$

We calculate the average probability that leakar + pe $i$ occurs in a time window for user $u$ :

$$
\lambda_{i}^{u}=\frac{1}{T} \frac{T-1}{T} \frac{0_{i}^{u, t}}{i} \text {. }
$$

Step 2. We then define the leakage probaw.'ity of that category as the previous probability averaged over all users of the same atege $y$ :

$$
\operatorname{Pr}_{i}^{\alpha}=\frac{\sum_{u=1}^{M_{\alpha}} \lambda_{i}^{u}}{M_{\alpha}}
$$

where $M_{\alpha}$ is the number of 'sers bu' nging to the category $\alpha$.

Step 3. The correspouding , rofile privacy leakage rate is calculated over all the users in one particular category $x$ as

$$
r_{i}^{\alpha}=\frac{\sum_{u=1}^{M_{\alpha}} \sum_{t=1}^{T} r_{i}^{u, t}}{\sum_{u=1}^{M_{\alpha}} \sum_{t=1}^{T} \delta_{i}^{u, t}} .
$$

The probability, ,ed ,rofile of a user category $\alpha$ then consists of $\operatorname{Prob}_{i}^{\alpha}$ and $r_{i}^{\alpha}$, which can be calculate $\iota$ base ' on the data from our experiments. We respectively name them as high probability $\left(\right.$ Pro $\left._{i}{ }^{\alpha}=037\right)$, medium probability $\left(\right.$ Prob $\left._{i}^{\alpha}=0.68\right)$, and low probability $\left(\right.$ Prob $_{i}^{\alpha}=$ 0.44) profil s.

Both type of ,rofiles quantify the users' privacy leakage characteristics: the leakage probability $t \cdot a b,{ }_{1} r o b_{i}^{\alpha}$ determine whether type $i$ privacy leakage happens or not in a time window, wh reas the average leakage rate $r_{i}^{u, t}, r_{i}^{\alpha}$ determine the number of type $i$ leakages in 
that time window should they happen at all. The profiles will be used in our arge-scale tracedrive studies in the Section 5 to facilitate the understanding of the user relationsh ${ }_{\iota_{r}}$ inference from the advertiser's point of view.

\section{Social Relationship Inference Leveraging Privacy Leakages}

In this section, we systematically study the consequence of the pri $\ldots$ leakages obtained by advertisers by applying the privacy leakage model to two human 1 . - bility traces. We build a simulator utilizing the privacy leakage inference framework $t$, gener, te connections between users based on both the leakage profiles derived from Section $1 a_{\iota^{\prime}}{ }^{+h^{\prime}}$ human mobility traces. The human mobility traces are used to discover connection: be wee । users in both an academia and a city environment.

\subsection{Methodology}

\subsubsection{Human mobility traces}

We use two human mobility traces: the MI's mace [18], and the Foursquare trace [19]. We choose these two traces mainly because ic ns in these traces come from different

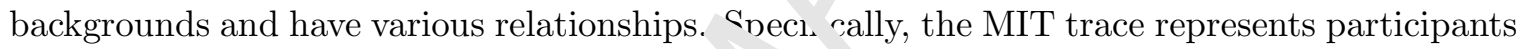
with similar background in an academia enviroı. ne..t, where user relationships mostly represent research colleagues, office staff and classmau In the Foursquare trace, participants have more diverse relationships; they may be collno oues, friends, and families in a city environment. The details of these two traces are intr fuced $l$ slow:

Foursquare Trace. Fours ${ }_{1}$ uare is 1 company that helps people share life experiences based on locations such as resta " ints This trace is generated based on tipping information collected from different ven _ _ in Los Angeles (LA). A tip in a venue shows that one participant has carried out some essential actıvities (like dinning and shopping) at that venue. There are 104, 478 tips left by $31, \cdot 44$, articipants in this trace. We choose 354 participants from the top 10 venues (which are all resta irants) to generate encounter events between participants based on the time of the $\iota_{\Perp_{1}}{ }^{n}$ ng $; .1$ a 21 day duration.

MIT Tracr. Thi trace is collected on MIT campus for 10 months by 107 participants with smartphont Ear 1 smartphone scans (using Bluetooth) and records nearby smartphones every five $\mathrm{n}$ inutes. The encounter happens when two participants are located in close physical proximity (e. sh swn in the Bluetooth scanned neighboring list in MIT Trace). And such an event is 'eh' eu as an encounter event. There are 97 participants with valid data including staffs and st dents. In our study, we use 21 days' data which includes 91 participants for social relationship inference. 


\subsubsection{Privacy Leakage Profile Population}

To understand the impact of user profiles, we repeat the study using hoth actı.eness and probability based profiles. When activeness based ones are used, each pa tici ant is assigned a randomly selected profile in the chosen category (i.e., active, regular anu 'nactı. ^). When probability based ones are used, each participant is assigned the same $\mathrm{r}$ ob hility profile (i.e., one of high, medium and low). When presenting our results, we will us + srms like "active users" or "users of medium probability" to (loosely) refer to particip unts a signed of the activeness or probability based profiles.

We then infer the relationships from the leakages over $o^{\prime}$ ser $u^{\prime} \mathrm{n}$ windows of different sizes (i.e., 7, 14 and 21 days). The inference is based on conr ntion $r^{\prime}$ rived from the leaked data. From real experiments with 10 participants having krown relc ionships, we find that different thresholds of connection counts in the observation w - dow wlould be applied to derive different relationships. In addition, depending on the mingtumds of the environment where the datasets are collected (e.g., MIT trace collected on can pus involves more academia relationships, and Foursquare trace collected in a city c $r$ ains even more diversity of relationships), the thresholds could also be different.

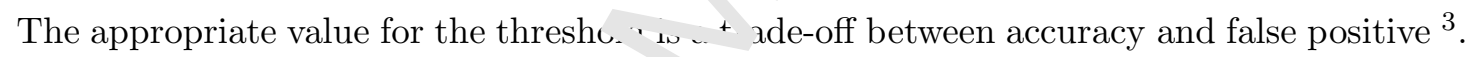
If the threshold is too high, we may miss some real relationships and have low accuracy; if the threshold is too low, we wil' "iden ify" nonexistent relationships and have high false positives. In our work, we respertive ${ }_{\perp}$, 1 : 3 days and 2 days for fact-based and intelligencebased relationships in both exr sim „nts and simulations. Such thresholds enable an advertiser to achieve over $90 \%$ inferenr $\_$accu , les for regular users with very small false positive rate, which is a good balance between the two.

Foursquare Trace In rder to apply privacy leakage profiles to this dataset, we generate encounter events between , articipants as follows: for a particular venue, we give a visiting duration with a rar tom lens 'h ranging from 30 minutes to 2 hours to each tipping user. In the overlapped perior $f$ the aration of two users, we generate encounter events with a fixed time interval of $30 \mathrm{~m}$ nutes ( .g., in an 1 hour overlapped period, we generate 2 encounter events). For each en' vunter event, we first find out corresponding 5-minute time windows. Then we flip a coin ith th privacy leakage probability in the users' profile (defined in Equation (2) or (7)) 1 nide whether privacy leakages should happen or not in that 5-minute time window.

\footnotetext{
${ }^{3}$ The accuracy and false positive rate are defined in Section 5.B.
} 


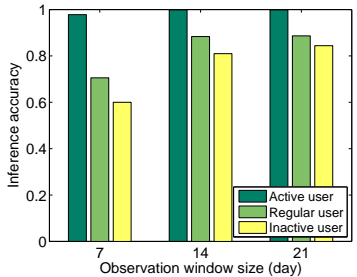

(a) Fact-based

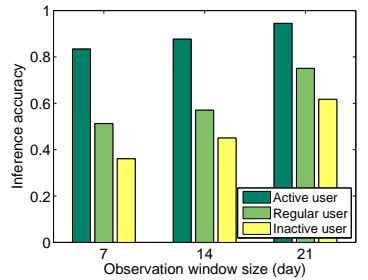

(b) Intelligence-based

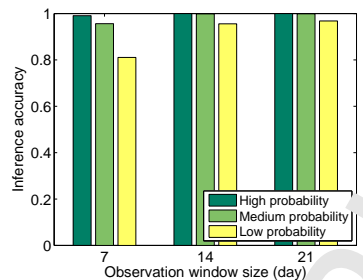

(c) Fact-basec

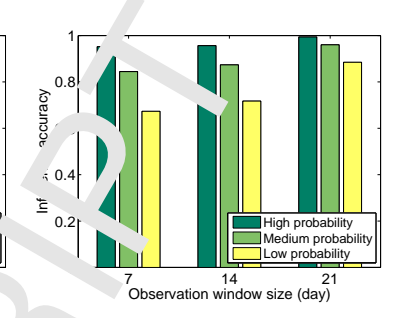

(d) Intelligence-based

Figure 9: Inference accuracy, MIT mobility trace, (a) and (b) are from the actı, ness based profiles using 3 days as the threshold, (c) and (d) are from the probability based profiles sing 2, ys as the threshold.

If they do, we use the privacy leakage rate defined in Equation ' 2 ) or (8) as the number of leakages revealed to the advertiser in that particular 5-min $1 \mathrm{te} . \mathrm{um}$ window. The leakages are used by the advertiser to derive connections and encounte evtuus to infer users' relationship.

MIT Trace. The MIT trace records encounter eve ts for e ich participant every 5 minutes. For each encounter event, we first find out the correspu ding 5-minute time window. Then for each of the two participants, we flip a coin with $\iota^{\circ}$ ? privacy leakage probability in his profile (defined in Equation 2 or Equation 7) to d : whether privacy leakages should happen or not in that 5-minute time window. If thoy $d c$ we use the privacy leakage rate defined in Equation 3 or 8 as the number of leakages reve. leu to the advertiser in that particular 5 minute time window. The leakages are used by $\because \cdots$ auvertiser to derive connections and eventually encounter events to infer users' relationship.

\subsection{Metrics}

In our evaluations, we study the inference accuracy of pairwise social relationship and the correlation between social commu itie, extracted based on the connections of users.

Inference accuracy. $\operatorname{Tr} \mathrm{s}$ : the ratio between the successfully inferred relationship pairs and all relationship pairs

Community correlaı $n$. This is the ratio between the common subjects within a community identified by our pri acy leakage inference framework and the total number of subjects within the community.

False positir, rate. This is the ratio between the number of mistakenly identified members of inferred comm nitr and the total number of members within the inferred community.

\subsection{Infere' se with Privacy Leakages}

\subsubsection{C mhination of Privacy Leakages}

As we i scussed in Section 4, an advertiser can utilize the temporal and spatial patterns of connections to infer users' relationship. There are multiple privacy leakages that can produce 


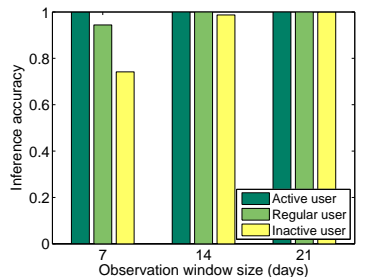

(a) Fact-based

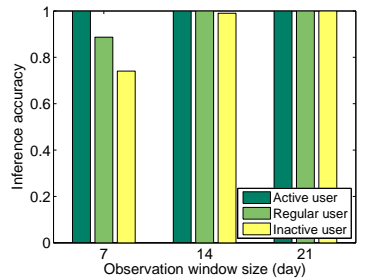

(b) Intelligence-based

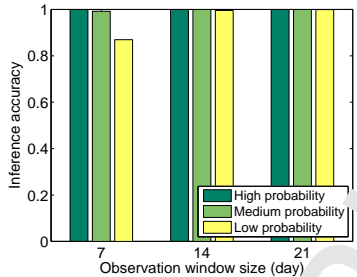

(c) Fact-basec

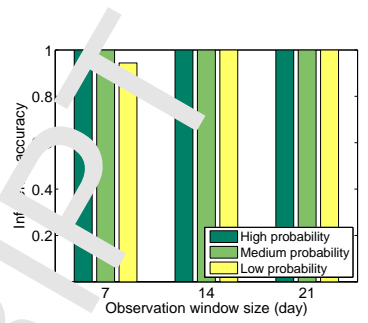

(d) Intelligence-based

Figure 10: Inference accuracy, Foursquare mobility trace, (a) and (b) are from i a activeness based profiles using 3 days as the threshold, (c) and (d) are from the probability based J ofiles u ing 2 days as the threshold.

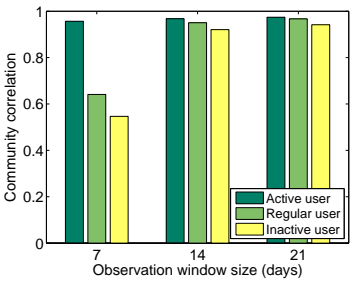

(a) Fact-based

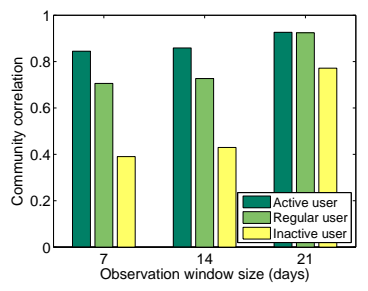

(b) Intelligence-based

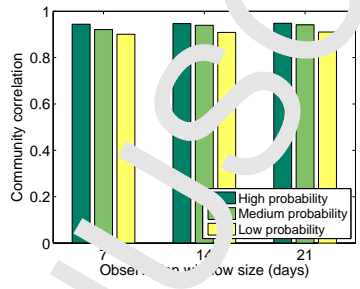

(c, Tract-based

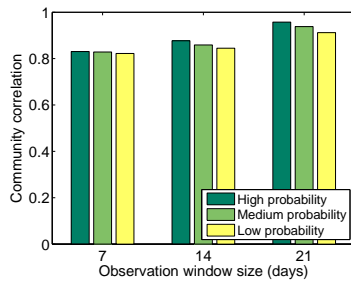

(d) Intelligence-based

Figure 11: Community correlation, MIT dataset, (a) and ( $u$, are from the activeness based profiles using 3 days as the threshold, (c) and (d) are from the probability rnced protı s using 2 days as the threshold.

connections between users. In this study we focus a the \{user identity, location \} combinations of most popular privacy leakages including $\mathrm{IN}_{\perp} \overline{\mathrm{I}}_{1}$, , hone number, GPS location, Wi-Fi AP list, and network-based location.

\subsubsection{Pairwise Social Relationship nfer nce}

Figure 9 compares the accuracy of $\mathrm{p}$ irwise social relationship inference (for both fact and intelligence based relation aips, by applying activeness and probability based profiles to the MIT trace under differe t siz ' i observation windows. We use 3 days and 2 days as the threshold for fact-basf $d$ als $^{\prime}$ intelligence-based relationship inference respectively, which is introduced in Section $\pm(\varepsilon$ me threshold applies hereafter). The fact-based relationship has greater threshold becaus seople having fact-based relationships are supposed to encounter each other more rer, ular.y than those having intelligence-based relationships (e.g., colleagues meet 3 days a week wh's riends meet 1 day a week).

From Figur, 9 (a) i nd (b) we observe that for most cases, an advertiser can achieve over $80 \%$ inferencr ccus wy for fact-based relationships with regular users, whereas it is around $60 \%$ for intellige 'ce-bası d relationships. We also observe that the inference accuracy decreases for less actim nsers, which is reasonable since less usage leads to less privacy leakages. In addition, for active ' ers, Figure 9 shows that the inference accuracy for both fact-based relationship and intelligence relationship is high (i.e., above $90 \%$ ). 


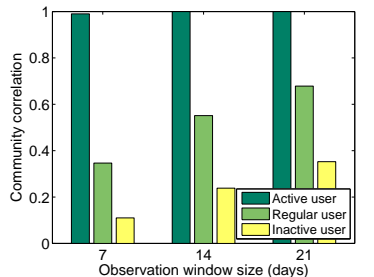

(a) Fact-based

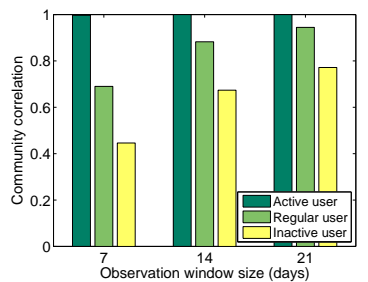

(b) Intelligence-based

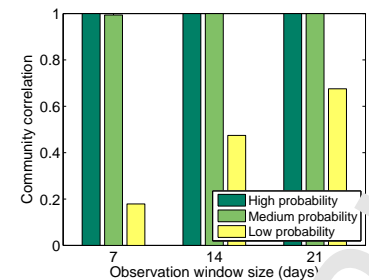

(c) Fact-basec

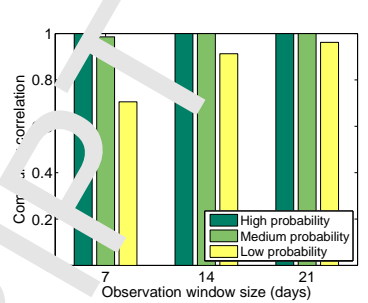

(d) Intelligence-based

Figure 12: Community correlation, Foursquare dataset, (a) and (b) are from the a veness based profiles using 3 days as the threshold, (c) and (d) are from the probability based profile using - days as the threshold.

Furthermore, we find that longer observation windows heln $i_{\nu_{\perp}}$ e the inference accuracy, especially when the privacy leakage probability is low. This is $r^{\prime}$ 'ser ed in the probability-based approach shown in Figure 9 (c) and (d). It is because a ' $n$ nge window helps the advertiser to accumulate more data, resulting in more connectic - to ide tifify users' relationships. Comparing Figure 9 (a) and (b) to Figure 9 (c) and (d) res, sctively, we observe that the inference accuracy of active users is similar to that of users ave the profile with a high leakage probability. Figure 9 (d) suggests that in order to $k_{L_{F}}$ tho inference accuracy of intelligence based relationship lower than $60 \%$, the user has ${ }^{+} \mathrm{o} k \mathrm{ke}$, his leakage probability smaller than low probability (i.e., 0.44).

Examining the Foursquare trace, we on arve much higher inference accuracy for both fact and intelligence based relationships. We show the results using privacy leakage profile with different activeness in Figure $10\left(a^{\prime}\right.$ and (b) For inactive users, the inference accuracy is about $70 \%$ for a 7 -day window, and it soes or $95 \%$ for the 14-day window. This is because users in the Foursquare trace encou ${ }^{2}{ }^{\circ}{ }^{2}$ eac'. other more frequently than those in the MIT trace. Thus, more connections can e discovered when the users have the same intensity of app usage, leading to a higher inference accu.acy. It is also the case when using the probability-based profile to infer pairwise rela ionship with the Foursquare trace (i.e., Figure 10 (c) and (d)).

\subsubsection{Social Comr uni+ Inference $^{\text {f }}$}

We next stud- how "a social community could be inferred by the advertiser using the privacy leakage profile. In particular, based on the inferred pairwise social relationships, a hierarchical iustermy algorithm [25] is applied to obtain the social communities of users with similar rela ionship, (e.g., collaborators, labmates, and classmates).

Cor minity Correlation. Figure 11 shows the community correlations calculated when applying $\mathrm{t}$. : activeness and probability based profiles on the MIT trace with three different observation windows. We observe that overall the community correlation is above $60 \%$ for 
Table 2: False positive rate for community correlation: MIT trace and Foursqu $\mu$ +race.

\begin{tabular}{|c|c|c|c|c|c|c|}
\hline & \multicolumn{3}{|c|}{ Fact-based } & \multicolumn{3}{|c|}{ Intelligence-based } \\
\hline & 7days & 14days & 21days & 7 days & 14days & 21 days \\
\hline \multicolumn{7}{|c|}{ MIT Trace } \\
\hline Active users & 0.035 & 0.022 & 0.016 & 0.149 & 0.129 & 0.098 \\
\hline Regular users & 0 & 0 & 0 & 0.065 & 0 & \\
\hline Inactive users & 0 & 0 & 0 & 0.056 & 0 & J \\
\hline High prob. & 0.069 & 0.054 & 0.052 & 0.17 & 0.145 & 0.17 \\
\hline Medium prob. & 0.045 & 0.041 & 0.039 & 0.104 & 0.093 & $0 . \mathrm{c}^{\mathrm{T}}$ \\
\hline Low prob. & 0.026 & 0.016 & 0.015 & 0.055 & $0.0 ?$ & $0 . \mathrm{C}^{257}$ \\
\hline \multicolumn{7}{|c|}{ Foursquare Trace } \\
\hline Active users & 0 & 0 & 0 & 0.019 & $0.07 \mathrm{c}$ & $0 . \Gamma_{0}^{0}$ \\
\hline Regular users & 0 & 0 & 0 & 0.081 & u. 661 & 0.052 \\
\hline Inactive users & 0 & 0 & 0 & 0.04 & $0 . \Gamma^{2}$ & 0.029 \\
\hline High prob. & 0 & 0 & 0 & 0.19 & .078 & 0.078 \\
\hline Medium prob. & 0 & 0 & 0 & $0.1 \diamond$ & $0 . C$ & 0.078 \\
\hline Low prob. & 0 & 0 & 0 & 0.099 & ר 64 & 0.058 \\
\hline
\end{tabular}

regular users. Furthermore, we observe that the co» munity correlation also increase with longer observation windows, especially for regular - ers and inactive users. This is also because longer observation windows help the advertise - a orregate more connections between users, which helps to more accurately identify their soc. 1 communities.

We present the community correlations $\iota^{f} \mathrm{v}^{+} \mathrm{h}$ fact based relationship and intelligence based relationship when activeness and pr'aninicy based profiles are applied to the Foursquare trace in Figure 12. We observe that the intelligence based community correlation is high for active users and regular users (i.e., ' ver 80\% on average). Similarly it is high for users with high probability and medium probab; ity pı f es (i.e., over 90\%), which is comparable to those of the MIT trace. However, the c $n \mathrm{~m}$ unit correlation of users having the fact-based relationship is much lower with inactive sers anu users with low probability profiles (i.e., ranges from $10 \%$ to $60 \%$ ) than that of the MIT tran. This is because participants in Foursquare data are from much diverse backgrou $\mathrm{d} \mathrm{i}$. the city environment and the locations are mostly restaurants which favors more to the in. "ligence-based relationship inference.

Discussion of $\mathbf{F}_{\mathbf{a}} \mathbf{I}, \mathbf{e} \mathbf{F}$ ositive. Table 2 shows false positive rate of community correlation for both $\mathrm{M}^{+}+$and $\mathrm{r}$ uursquare traces. Overall, the false positive rate is very small. In addition, larger bserve ion window has smaller false positive rates. This indicates that longer observation winder size has more information and can improve the inference performance. Furthermore we o serve that the Foursquare trace has much lower false positive rate than the MIT $\operatorname{tra}{ }^{2} \mathrm{e} \cdot \mathrm{hl}: \mathrm{s}$ is because the Foursquare trace has more connections, thus leads to lower false positi $、$ rate.

To briefly summarize the major findings, 1) the advertiser can infer users' social relation- 
ships at high accuracy, e.g., over $90 \%$ on average for active users and over $70 \%$ on average for regular users; 2) the advertiser can also infer a significant portion of users smmunity relationships, e.g., over $90 \%$ on average for active users and over $60 \% r \mathrm{a}$ av rage for regular users, which reveals common interests or activities among users not ocess - wily with direct interactions. The results from the simulation confirm the findings or our real experiments with the relatively smaller number of participants and shorter terı.

\section{Privacy Leakage Visualization}

Since advertisers can infer users' relationships and comr w..ities ıom the privacy leakages, a visualization tool that presents such leakages in real tim $r$ ay b lp users better adapt their app usage. Such tool would reveal the detailed temporal aı ' spatial characteristics of privacy leakages to complement existing works, such as the cre 'rdsov cing-based privacy setting tool proposed in [13].

\subsection{Tool Features}

We present our privacy leakage visualization $t_{0}, 1$ that can display the leakage statistics at different levels of granularity. Existing visuc'k ${ }^{\prime}+t_{\text {io. }}$ tools usually address each app individually or focus on a specific type of priva . loak $k_{c}$ res $[26,27]$. For example, by displaying the privacy data that an app intents to access, ex ' ing work allows the user to block such privacy leakage [20] or helps her obfuscate ${ }^{\prime} \mathrm{e}$ pı racy information $[11,12]$.

In contrast, our tool provides spe ${ }^{\text {ial- }}+$ mporal visualization of the leaked private data at multiple levels of granularity, ; 1 clv ing the global level, whereby the statistics over all apps and destinations (i.e., advert sers, * ummarized; the destination level, whereby the privacy leakages received at each $\mathrm{d}$ stı. ${ }^{+}$ion are shown; and the app level, whereby the leakage can be displayed per app and p r u 'er's location. Such comprehensive statistical visualization avoids presenting low-level raw : ra to users who are mostly not tech-savvy and cannot make sense out of the raw data It acilitates a better understanding of the privacy leakage and provides users opportunities to ${ }^{\mathrm{l}} \mathrm{i}$. st her app usage pattern to control such leakage.

Our tool uti zes Ta itDroid [3] (with Android OS 2.3.4) to capture the leakage information. The tool rur - n vanground and logs the details of each privacy leakage event including the privacy typ ?, the ( ontent of leaked data, the app name, the destination, and a timestamp. It consirts of uve main components: Global Viewer, Map Viewer, Statistics Viewer, AppDestinatic Viewer, and App Leakage Warning. The main components and the flow of the tool are depicted in Figure 13. 


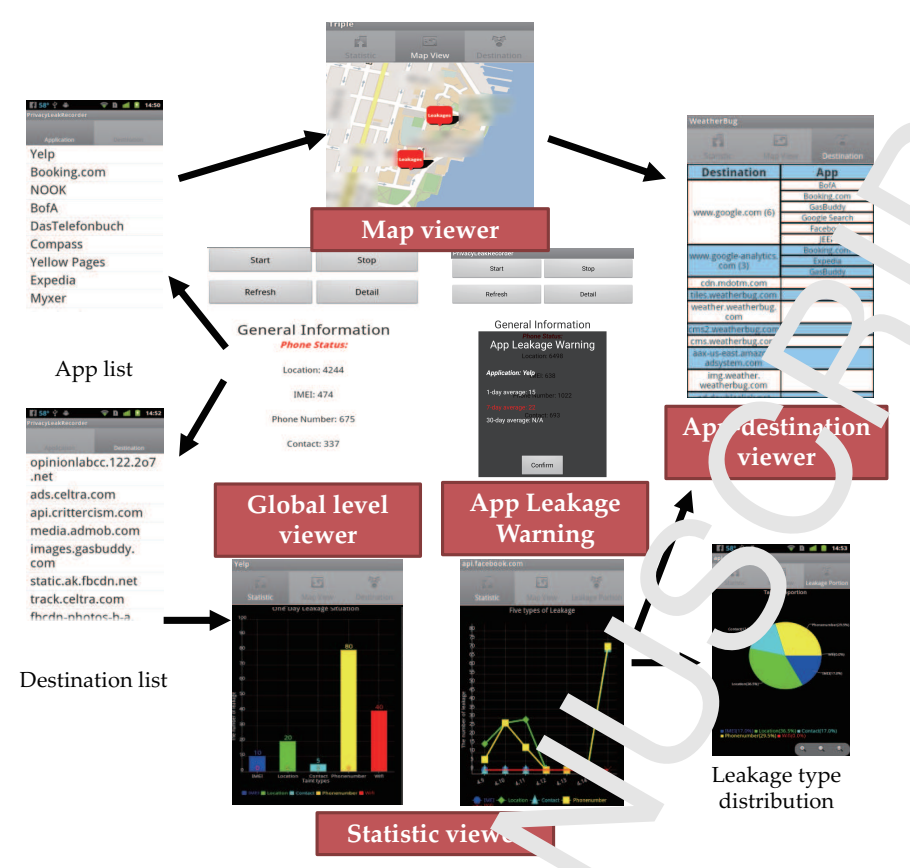

Figure 13: GUI flow c …-icinalization tool.

Global Viewer. It presents the overall $>$ rivac ` leakages of the mobile device by aggregating the leaked information from all apps in a ı ser lefined time period.

Map Viewer. It shows a detailed spaı. ' distribution of leakage information including the type and occurred time over a map.

Statistics Viewer. It provid s temp ral statistics of different types of privacy leakage using scatter plots and pie chart ${ }^{\dagger}$.

App-Destination Viewe . t displays the connection between apps and destinations: what private data is leaker' ${ }^{c_{r}}$ om which apps to the same destination. The destination can integrate the data from all these apps to infer knowledge about the user.

App Leakage $\mathbf{W}_{\boldsymbol{i}} \cdot \mathbf{n n i}^{\mathbf{r}} \mathbf{g}$. The visualization tool dynamically sends active warnings to users based on the a eraore te. kages of apps. The warning message includes a summary of the average leakages an ${ }^{n}{ }^{n}$ ovir es a shortcut link to the detailed statistics of the privacy leakages of the highlight $d$ app

\subsection{Global Viowe.}

The vis talizati n tool starts with the Global Viewer, which shows the total numbers of privacy leakagto ur each data type (i.e., Phone number, IMEI, Location, Contact list, and WiFi AP list, ? , illustrated in Figure 13) over a user-defined time period or the total history of the phone usage. The Global Viewer has two branches: App List or Destination List, which show 


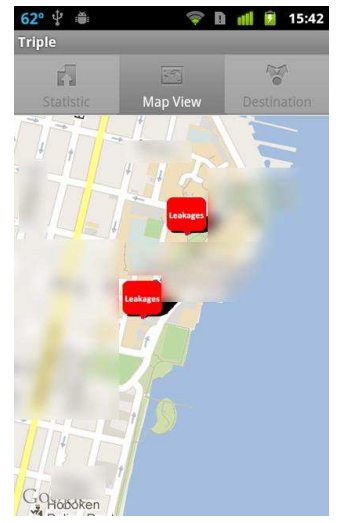

(a) Two places of multiple leakages

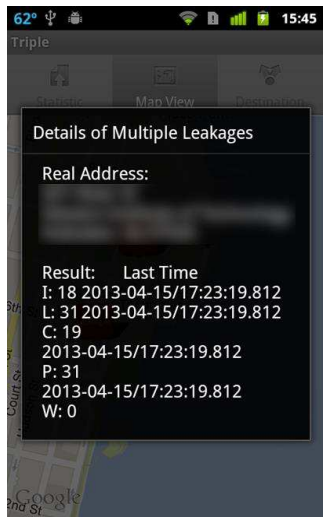

(b) List of leakages with detailed information

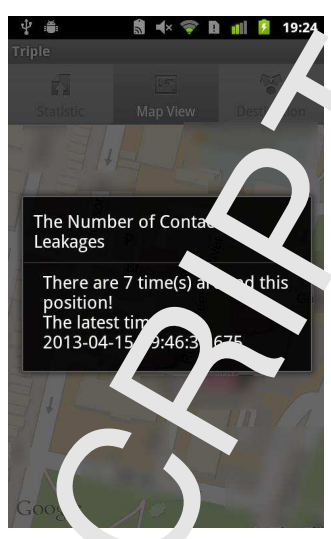

1; , Selected one deta led leakage

Figure 14: Screenshots of multiple privacy leakages founa be ! .ed at different locations.

the lists of apps that leak private data and destinatic 's that eceive it (shown in Figure 13). By observing the privacy leakage counts per app, a use can easily tell which app has the most frequent leakage of sensitive data.

\subsection{Map Viewer}

The Map Viewer shows the physical loc tions vhere the private data is leaked. The Map Viewer can be accessed from the App list or the De iination list, which will show on the map all the leakages from one app or to one destina 'nn (e.g., Figure 14 (a)). The user can also further

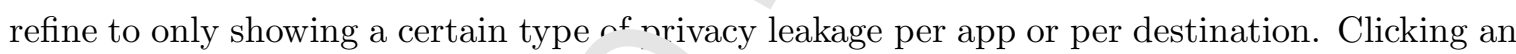
icon will display the details of priv cy leakc ye at that location. Figure 14 (b) and (c) illustrate the detailed information of all ty es of pis acy leakages and the detailed information of contact information leakage, respective, We ranslate the GPS coordinates into the corresponding physical address using Goo',- Maps API for easy understanding to the user.

One implementation imre is how to display the privacy leakage events when a lot of them occur around the sam lor tion. This happens when the user stays at the same location for some time durin', which _..ultiple leakage events of different types can happen. The Map Viewer combines eve +, of ne same privacy type and shows them as one icon on the map. It determines whe ner tv $>$ locations can be combined if their distance is less than a threshold.

Different typ of $\mathrm{p}$-vacy leakages are displayed in icons of different colors. When too many events occv at oi location, a single red icon is shown to avoid crowdedness. Figure 14 (a)

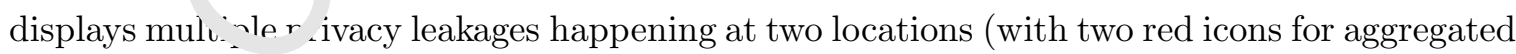
privacy 1 aks jeoj. Clicking on the icon will display the detailed information about the privacy leakages, su 3 as the physical address, historical counts of different types of privacy leakages, and the most recent leakage time (shown in Figure 14 (b)). Clicking on an icon of a specific 


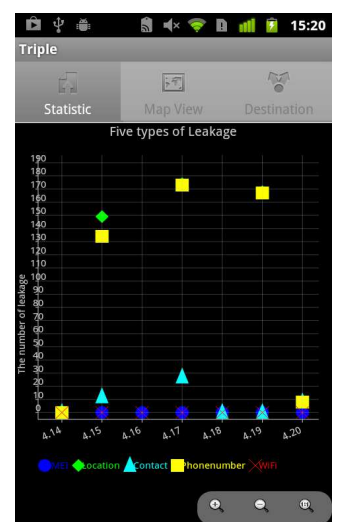

(a) Privacy sinks

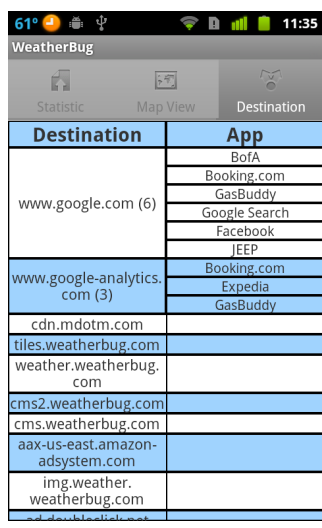

(b) Other apps sharing the destination

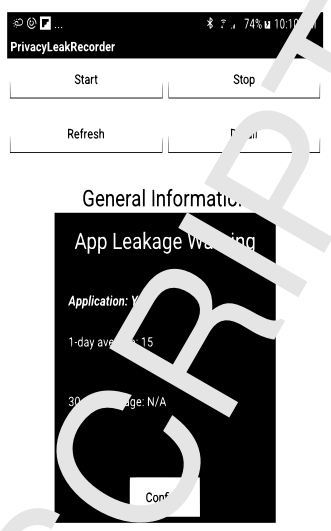

(c) A .. Leakage Warn-

in message

Figure 15: Screenshots of (a) \& (b) App-Destination View anc ', App Leakage Warning.

type of privacy leakage will display the historical coun ${ }^{*}$ s of th th type of leakage and the most recent leakage time (shown in Figure 14 (c)).

\subsection{Statistics Viewer}

The Map viewer summarizes the spatial $d_{1}+\operatorname{tri}^{\prime} u_{\text {uron }}$ of privacy leakages. The Statistics Viewer reveals the temporal patterns of each , ne o ' 'eakage events such as IMEI, GPS location, Wi-Fi Access Point SSID list, contact lis ${ }^{+}$and ${ }_{1}$ hone number. The Statistics Viewer plots the numbers of different types of privacy leakag over a time window, whose sizes are 1-day, 7day, and 30-day to show the leakage pa 'rn in both short-term and long-term. Figure 15 (a) depicts the statistics over a 7-day, oriod Leakage patterns in fine-grained time slots (e.g., one-hour) are also available in $\mathrm{f}$ r $g$ aphs. These statistics potentially can help a user identify apps leaking private data agg ess. ^ly ind alter his/her usage patterns of these apps (e.g., use them less frequently or sto, a, ngether).

\subsection{App-Destination V ewє .}

The App-Destination : : ower presents how much private data each destination can obtain across multiple apr, w ich determines the scope and depth of the knowledge one advertiser may infer about th 2 ust. Figure 15 (b) presents an example of App-Destination Viewer for the app Weath rBug. The screen is separated into two columns: the left column lists the destinations $u$ whici the selected app has sent private data, and the right column shows other apps that a so senc private data to the same destinations.

\subsection{Apt Ler uje Warning}

In addi on to providing various interfaces for users to have the complete and detailed picture of the privacy leakages in their smartphone use, we design an App Leakage Warning 
mechanism, which can actively send a warning message to users about a par 'cular app when the average leakages of the app have crossed a certain threshold. Figure 15 (c) p esents an example of the App Leakage Warning message for the app Yelp. The nes age includes the name of the app, average leakages in different scales (i.e., 1-day, 7-c v, aı 30-day). The average leakage crossing the threshold would be highlighted in red. ', st. s couıd directly go the detailed statistics by tapping the name of the app in the message.

\section{Discussion}

More Types of Private Data. There are potentially $\mathrm{c}^{+1}$ or t ty $y_{\mathrm{F}}$ of private data available to advertisers. For example, Google has the access to its a ch t rms and histories of many users. Although we do not find the 190 apps studied lea ing rext message logs, audio and video data, illegitimate access and disclosure of sucı inform ation are not impossible. Our current study is based on the combination of most bas privacy leakages (i.e., identities and locations). Contact list carries important informa inn. For example, a certain relationship most likely exists when two users share many c um. - ontacts. Unfortunately, because we do not have the contact lists of the subjects of t'o twe mobility traces, we are not able to evaluate its impact on relationship inference. Studying the impact of more types of private data would be interesting future work.

Large Scale Evaluation. We am aware that our experiments (due to limited available manpower) may cause bias to our ; rivacy 1 akage profiles and the evaluation may not cover all privacy leakage patterns in the $r$ al worlu In particular, the ten volunteers in our experiments are most graduate students an' nly cwo of them are family-related. We mainly use this experiment to demonstrate ' $\therefore$ possibility of revealing users' social relationships by using the spatial and temporal information of the privacy leakages in their smartphone use. In addition, we are also aware tha our experiments may be limited to independent app behaviors due to the limited numb or of a $\mu_{\mathrm{s}} \mathrm{s}$ used in our real experiments. Involving more apps running simultaneously in $t_{1}$, sam phone would better reveal the privacy leakages of apps having certain depende cies. 'ncreasing the number of participants and apps in real experiments and building more sc. histir tted privacy leakage profiles are our future exploration as well.

Insuffi ient Metadata. Our evaluation is constrained by the availability of metadata descriptions i do asets. Neither the MIT nor Foursquare data has sufficient annotation to different, te we relationships among users at fine granularity desired by us: colleague, collaborator, class nate, friend, and family. In lieu of that, we have to utilize the most commonly used technique for detecting social communities (i.e., hierarchical clustering algorithm [25]) and 
use the results as the base of comparison, which is a common practice in s' cial relationship research. In the future we hope such metadata would be made available when peor 'e conduct such experiments.

More Advanced Inference Algorithms. The thresholding als rith.t that we use to infer relationships is based on quite simple heuristics. With the ar ulc bility of large amount of data, advertisers can use more advanced inference algorithms, e.s 'y utilizing data mining techniques. In addition, certain relationship may have similar spatia ${ }^{1}$ and temporal patterns that may be hard to distinguish by using the naive algorithm, su $h$ as $f$ mily and friends if they live together. However, it may be possible to reveal the dif əren $u$ based on even finer-grained analysis. For example, family members usually have bre ${ }^{1}$ fast an ${ }^{\prime}$ dinner together at the same time while friends may have totally different schedules. We , re aware of this limitation and

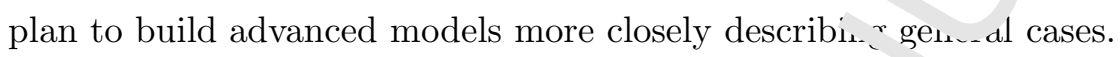

Time Guidelines from the Visualization $m$. . . v ur visualization tool provides spatial temporal statistics of privacy leakages, which gives the $\mathrm{u}$ or better ideas of the scope and degree of privacy loss. Ideally, based on the type, destil + . on, location and frequency of the leakages, an inference algorithm can be used to estin, 'u how much of the user's relationships can be derived. The tool would then be able t w.ri, a app usage guidelines in real time, such as stopping using certain apps for some time, wheı certain conditions are triggered (e.g., a certain fraction of a kind of relationship is e pose ?. This estimation requires certain knowledge about the usage patterns of other related us ' ', vailable to advertisers but not to us without a wide enough installation base of the $\mathrm{oo}^{\mathrm{l}} \mathrm{Hc} \mathrm{v}$ to make educated guesses on such knowledge would be one interesting research ir sue.

Emerging Visualiza.ion - $\boldsymbol{n}$ ol. We are also aware there are several visualization tools has been proposed rece tly $[28,29,30]$. XDroidet al. [28] is a machine-learning based tool that can detect malware 'ased on apps' behaviors. Liu [29] proposed a personal privacy assistant that can $r$ aild per aission profiles based on people's choices and provide permission

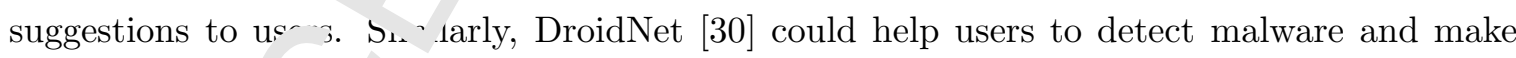
decisions on en bling c ops' permissions based on the suggestions from experts through the cloud servicr. However, all these tools are focusing on permissions setup and do not reveal real-time pi vacy lf $x$ kages and the more serious consequence of privacy leakages integrated at the sam rnctination. 


\section{Related Work}

Enck et al. [10] are the first to conduct permission analysis to identify $r$ ngerous functionalities using Android permissions, such as tracking user or voice eavesdr npi g. They propose Kirin, which performs inspections on Android API permissions durin ${ }_{\circ}$ the upp installation time. They examine 311 top free applications and identify severa' qu ${ }^{c+}{ }^{+}$onable applications sending out users' private information. Barrera et al. [5] perform per ${ }_{\perp}$ ssion analysis of the top 1,100 free applications and report many applications request $r$ nly a ^ nall set of permissions. Felt et al. [6] study 100 paid and 856 free applications from no A . tdroid Market and find INTERNET permission is the most frequently requested. $\mathrm{Tr}-\mathrm{y}$ ater propose Sowayway [9] to detect over-privilege in applications and report $10 \mathrm{m \iota}{ }^{+}{ }^{+}$cor ${ }^{*}$. on unnecessary permissions. Taylor et al. [35] analyze a privilege escalation attack, where he third-party libraries can get access to sensitive data from devices using intra-libra. "r conusion. And they prove that several popular libraries facilitate this attack by coli $1,1 n g$ enough sensitive data. Pennekamp et al. [36] provide a survey, which reviews the nthods uhat have been proposed to check the applications permission and their access to sensi $\cdot$ e information.

Static analysis analyzes the code of applici ' 10.7 to infer what can happen to users' security. For example, PiOS [31] analyzes compile; uvj tive-C code to identify information leaks on the iOS platform, whereas ComDroid [32] uses disassembled DEX bytecode to identify vulnerabilities in Intent communication $r$ etween applications. Enck et al. further propose the ded decompiler [34] to reverse Andrc d app ${ }^{\prime}{ }^{\circ}$.tions to Java code for security analysis. FlowDroid [37] can detect private data le ks; 1 A' droid apps by performing a data flow analysis, which becomes a highly popular $\mathrm{s}^{\dagger}$ thic ana.ysis framework. DroidSafe [38] uses the static analysis on Android information flow to 1 - ort the potential leaks of sensitive information.

Less work has been don in dynamic analysis. TaintDroid [3] tracks the flow of privacysensitive data througr thiru . arty applications. It detects when sensitive data leaves the system via interfaces such эs etw rk connections. Agarwal et al. [13] propose ProtectMyPrivacy

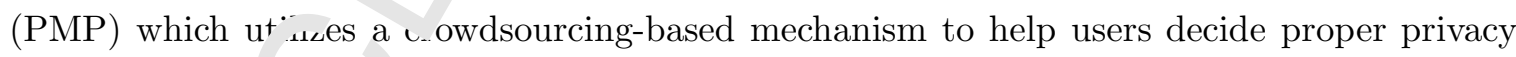
settings for iOS ıpps, $\mathrm{s}$.ch as denying access to private data or substituting anonymized data. He et al. $\left[3^{r}\right.$ prorose a privacy leakage analysis framework for third-party libraries in real time using both c rmbin $d$ static and dynamic Xposed methods. Chen et al. [40] propose a method called $\mathrm{E}$.nnid which gets the advantage of both static and dynamic analysis methods to identify the private data leakage. Using the combination of both static and dynamic analysis, HybriDroid can detect data leakage for both inter and intra-app communications with the 
high accuracy. Brandtzaeg et al. [41] also apply the dynamic analysis to nalyze the data flow and measure activity in the apps over a long time, which strengthens the unu rstanding of the complexity of privacy issues in mobile apps. Our work takes $\downarrow \mathrm{di}^{\top}$ erent viewpoint by systematically analyzing what privacy-sensitive information the ad. $\neg$ rtist , san collect and aggregate at run-time from multiple apps and infer the social relatic ssi $\mathrm{p}$ of a user. We utilize TaintDroid as a tool to track and log the run-time privacy leakage $" \backsim n$ apps.

A few app tools are developed to provide a user with the $\mathrm{F}$. Ivacy 'eakage information [11, $7,8]$. However, the information is provided in low-level and $r_{c} \times$ dat format based on each app, which only gives the user a narrow view of the priva y le su ge on a per-app basis. Our visualization tool seeks to provide a multi-level statisti al view of privacy leakage and gives the user a better understanding of the implication of leaked $\mathrm{p}$ ivate information.

Finally, some work develops smartphone platfor - bas a privacy protection mechanisms. Ongtang et al. propose Saint [26] and Porscha $\left[2^{-1}\right.$, w w w inding the functionality of the Kirin system to allow runtime permission inspection bv dehı. 'ng runtime policies. Bugiel et al. propose XManDroid [42] to mitigate permission priv 'e e escalation attacks in Android by tracking communication between components in differ $\tau_{u}$ applications. To prevent smartphone applications from leaking phone identifiers and . " provide empty, fake or anonymized information to applications. AppFence [20] builds on top of Taintdroid to actively block net ork $u_{\text {. }}$ nnsmissions containing user-defined sensitive information, which should be used or the tev ce only. Our work focuses on a different aspect of gaining a systematic understar tins of the social relationship inference consequences from the privacy leakage by an adver iser. $\sim$ h understanding may motivate the user to adjust app usage pattern or adopt dés nse ${ }_{\text {. }}{ }^{\circ}$ chanisms to control the sensitive data leakage.

\section{Conclusion}

Privacy leakage ', y smar, shone apps has attracted significant research efforts in recent years. The commun to has proposed various defense mechanisms, from permission management, code ana'ysis, t $\mathrm{n}$ obfuscated data. Nevertheless, the characteristics of apps' run-time privacy leakage ohavi $r$ is still not well investigated, and the consequences of such privacy leakages ha re not attracted much attention. This paper serves as the first step towards a comprehensı v erstanding of the advertiser's perspective. In particular, we seek to discover what an adv suser can infer about users' social and community relationships by combining private data 'rom many apps. Our analysis on the run-time privacy leakage behavior of nearly 200 most popular apps from 19 categories of Google Play shows that dominant advertisers 
can easily gather data from many apps. We propose a privacy leakage inff ence framework that describes a general method for inferring users' social and community relation. hips. Our experimental study over one month demonstrates that an advertiser cr 1 in $ə r$ r $90 \%$ of users' social relationship correctly using simple heuristics. This observation . furı. ar confirmed by human mobility trace driven studies of two large scale data sets. ve finalıy build a visualization tool that can capture and display the spatial-temporal stau. +i is of privacy leakage to different advertisers in real time. We hope our work will eventı ally le d to a complete picture of the advertiser's perspective.

\section{Acknowledgement}

This work is supported in part by the National Scien Foundation Grants SES1450091 and CNS1217387.

[1] M. C. Grace, W. Zhou, X. Jiang, A.-R. Sades · unsare exposure analysis of mobile in-app advertisements, in: Proceedings of the fif ${ }^{\imath}$ ACM conference on Security and Privacy in Wireless and Mobile Networks, 2012.

[2] R. Stevens, C. Gibler, J. Crussell, .T. Erı kson, H. Chen, Investigating user privacy in android ad libraries, IEEE Mobile Secu ${ }^{\circ}{ }^{+} \mathrm{v}$ Technologies (MoST).

[3] W. Enck, P. Gilbert, B.-G. Chv ., L. F. Cox, J. Jung, P. McDaniel, A. N. Sheth, Taintdroid: an information-flow trackins sysu $n$ or realtime privacy monitoring on smartphones, in: Proceedings of the 9th $\mathrm{U}^{\mathbb{S}} \mathrm{e} \mathrm{N}^{\top} \mathrm{X}$ cr aference on Operating systems design and implementation, 2010.

[4] A. P. Felt, E. Ha, S. _elman, A. Haney, E. Chin, D. Wagner, Android permissions: User attention, compre $\eta^{\prime}$ on, and behavior, in: Proceedings of the Eighth Symposium on Usable Privacy ind Secu ity, 2012, pp. 1-14.

[5] D. Barrera, "r G. ' tyacik, P. C. van Oorschot, A. Somayaji, A methodology for empirical ana vsis of sermission-based security models and its application to android, in: Proceer' ins of the ACM conference on Computer and communications security, 2010.

[6] A. P. Ft. $K$. Greenwood, D. Wagner, The effectiveness of application permissions, in: Wet 'p's, 2011. 
[7] X. Wei, L. Gomez, I. Neamtiu, M. Faloutsos, Profiledroid: multi-laye profiling of android applications, in: Proceedings of the 18th annual international conference on Mobile computing and networking, 2012, pp. 137-148.

[8] Lamian, LBE Privacy Gurad, https://play.google.com /store/apns/.

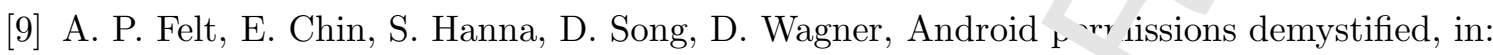
ACM CCS, 2011.

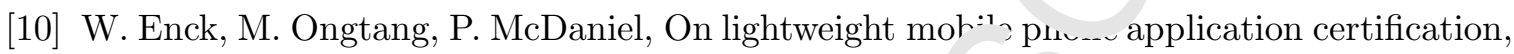
in: ACM CCS, 2009.

[11] A. R. Beresford, A. Rice, N. Skehin, R. Sohan, Mockdrol t trading privacy for application functionality on smartphones, in: Proceedings $0^{2}$ the $1^{2+1}$ Workshop on Mobile Computing Systems and Applications, 2011, pp. 49-54.

[12] Y. Zhou, X. Zhang, X. Jiang, V. W. F. i- Taming information-stealing smartphone applications (on android), in: Trust an ` Tru worthy Computing, 2011.

[13] Y. Agarwal, M. Hall, Protectmypr devices using crowdsourcing, in: MobiSy, 2013.

[14] M. Nauman, S. Khan, X. Zhar s, Ape ' extending android permission model and enforcement with user-defined runt me cor- raints, in: Proceedings of the 5th ACM Symposium

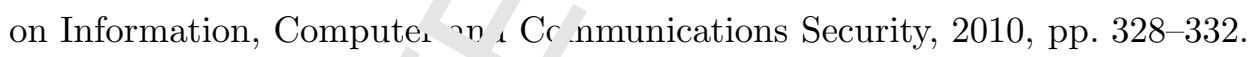

[15] K. W. Y. Au, Y. F. 7nou, 7. Huang, D. Lie, Pscout: analyzing the android permission specification, in: Ar $v \mathbf{T}$ TCS, 2012.

[16] C. Wilson, B. P je, A. `ala, K. P. Puttaswamy, B. Y. Zhao, User interactions in social networks and $a$ ir $1 \mathrm{mr}$.cations, in: Proceedings of the 4th ACM European conference on Computer s, stems, ¿J09, pp. 205-218.

[17] A. Crup tun fll Facebook, and Good Riddance, http://abcnews.go.com/ABC_ Univis $ว$ /qui ing-facebook/story?id=18668978\#.UYVrQ7W854I.

[18] N. as: 1. S. Pentland, CRAWDAD trace set mit/reality/blueaware (v. 2005-07-01), Downı ad from http://crawdad.cs.dartmouth.edu/mit/reality/blueaware (Jul. 2005). 
[19] J. Bao, Y. Zheng, M. F. Mokbel, Location-based and preference-aware ecommendation using sparse geo-social networking data, in: ACM SIGSPATIAL GIS, 2012, pr. 199-208.

[20] P. Hornyack, S. Han, J. Jung, S. Schechter, D. Wetherall, These art 't the droids you're looking for: retrofitting android to protect data from imperious applu tions, in: Proceedings of the ACM conference on Computer and communicatior, ser us , ACM, 2011.

[21] S. Thurm, Y. I. Kane, Your Apps Are Watching You, http://online.wsj.com/article/SB1000142405274870469400 ‘ 5760`J083703574602.html.

[22] D. Knoke, S. Yang, Social network analysis, Vol. 154, Sa' „, 208.

[23] P. Hui, E. Yoneki, S. Y. Chan, J. Crowcroft, Distribute ${ }^{1}$ community detection in delay tolerant networks, in: ACM MobiArch, 2007.

[24] Google.com, Configure access poinu with Google Location Service, http://support.google.com/maps/?hl=en.

[25] M. E. Newman, Detecting community … ^tu. ? in networks, EPJ B 38.

[26] M. Ongtang, S. McLaughlin, W. Enc. r. $\therefore$ Daniel, Semantically rich application-centric security in android, Security and Communication Networks 5 (6) (2012) 658-673.

[27] M. Ongtang, K. Butler, P. Mc 'aniel, 'orscha: Policy oriented secure content handling in android, in: Proceedings of ihe 26th Annual Computer Security Applications Conference, 2010 .

[28] B. Rashidi, C. Fung, E. Bu. +ino, Android resource usage risk assessment using hidden markov model and snli e learning, Computers \& Security 65 (2017) 90-107.

[29] B. Liu, M. S. A ıdersen, F. Schaub, H. Almuhimedi, S. A. Zhang, N. Sadeh, Y. Agarwal, A. Acquisti, Fon m recommendations: A personalized privacy assistant for mobile app permission , in: S. 'mposium on Usable Privacy and Security, 2016.

[30] B. Rast zui, C. rung, A. Nguyen, T. Vu, E. Bertino, Android user privacy preserving throug crowd sourcing, IEEE Transactions on Information Forensics and Security 13 (3) $\left(20^{+} \cap\right), 773-787$.

[31] M. Ege , C. Kruegel, E. Kirda, G. Vigna, Pios: Detecting privacy leaks in ios applications, in: NDSS, 2011. 
[32] E. Chin, A. P. Felt, K. Greenwood, D. Wagner, Analyzing inter-applica 'on communication in android, in: Proceedings of the 9th international conference on Mobis systems, applications, and services, 2011, pp. 239-252.

[33] L. Lu, Z. Li, Z. Wu, W. Lee, G. Jiang, Chex: statically vetting androiu nps for component hijacking vulnerabilities, in: ACM CCS, 2012.

[34] W. Enck, D. Octeau, P. McDaniel, S. Chaudhuri, A study , and”oia application security, in: USENIX security, 2011.

[35] V. F. Taylor, A. R. Beresford, I. Martinovic, Intra-lik ar ${ }_{4}$ co usion: A potential privacy nightmare on smartphones, arXiv preprint arXiv:17uc ${ }^{135} 3 \mathrm{u}$.

[36] J. Pennekamp, M. Henze, K. Wehrle, A surves on L- volution of privacy enforcement on smartphones and the road ahead, Pervasjm nnd * sbile Computing.

[37] S. Arzt, S. Rasthofer, C. Fritz, E. Bodde. : Dartel, J. Klein, Y. Le Traon, D. Octeau, P. McDaniel, Flowdroid: Precise contert, flc r, field, object-sensitive and lifecycle-aware taint analysis for android apps, Acm Sig̨ 'an Notices 49 (6) (2014) 259-269.

[38] M. I. Gordon, D. Kim, J. H. Perkins, L. `ilham, N. Nguyen, M. C. Rinard, Information flow analysis of android applice sons :n droidsafe., in: NDSS, Vol. 15, 2015, p. 110.

[39] Y. He, B. Hu, Z. Han, Dyn mic pı ıcy leakage analysis of android third-party libraries, in: Data Intelligence and ' r rity (ICDIS), 2018 1st International Conference on, IEEE, 2018, pp. 275-280.

[40] H. Chen, H.-f. Leu ๖, 3. Han, J. Su, Automatic privacy leakage detection for massive android apps via a $\ldots$ el hybrid approach, in: Communications (ICC), 2017 IEEE International Confe encr on. IEEE, 2017, pp. 1-7.

[41] P. B. Bran «'zaeg, +.. Pultier, G. M. Moen, Losing control to data-hungry apps: A mixed-metı ds ap roach to mobile app privacy, Social Science Computer Review (2018) 089443 ' 318777706.

[42] S. Bugieı, _. Davi, A. Dmitrienko, T. Fischer, A.-R. Sadeghi, Xmandroid: A new androic r slution to mitigate privilege escalation attacks, Technische Universität Darmstadt, Technic..l Report TR-2011-04. 